\title{
Oral Health Status and Need for Oral Care in an Aging Population: A Systematic Review
}

\author{
Dorina Lauritano ${ }^{1, *,+}$ (D) Giulia Moreo ${ }^{1, \dagger}{ }^{,}$Fedora Della Vella ${ }^{2}$, , Dario Di Stasio ${ }^{3} \mathbb{C}^{\text {, }}$ \\ Francesco Carinci ${ }^{4}$, Alberta Lucchese ${ }^{3}$ and Massimo Petruzzi ${ }^{2}$ (D) \\ 1 Department of Medicine and Surgery, Centre of Neuroscience of Milan, University of Milano-Bicocca, \\ 20126 Milan, Italy; moreo.giulia@gmail.com \\ 2 Interdisciplinary Department of Medicine, University of Bari, 70121 Bari, Italy; \\ dellavellaf@gmail.com (F.D.V.); massimo.petruzzi@uniba.it (M.P.) \\ 3 Multidisciplinary Department of Medical and Dental Specialties, University of Campania-Luigi Vanvitelli, \\ 80138 Naples, Italy; dario.distasio@unicampania.it (D.D.S.); alberta.lucchese@unicampania.it (A.L.) \\ 4 Department of Morphology, Surgery and Experimental medicine, University of Ferrara, 44121 Ferrara, Italy; \\ crc@unife.it \\ * Correspondence: dorina.lauritano@unimib.it; Tel.: +39-3356790163 \\ + These authors contribute equally to this work.
}

Received: 3 November 2019; Accepted: 10 November 2019; Published: 18 November 2019

check for updates

\begin{abstract}
Background. The world population is aging. This phenomenon is accompanied by an increase in the number of elderly with dementia, whose oral hygiene care is a challenge. Objective. This paper presents a literature review of oral health status and the need for oral care in people with dementia, as compared to people without dementia and also of the relationship between periodontal disease and cognitive impairment. Methods. A systematic review was conducted in PubMed, CINAHL, and the Cochrane Library. Fifty-six articles met the inclusion criteria and were consequently included for quality assessment and data extraction. Results. No significant differences were found between both groups with regard to the number of present teeth, DMFT Index, edentulousness/use of denture, and orofacial pain. Coronal/root caries and retained roots were more common in people with dementia than in those without dementia. Most of the participants with dementia presented gingival bleeding or inflammation and they suffered from the periodontal disease more than people without dementia. Conclusions. Poor oral health is a common condition among the elderly with dementia. The education process of caregivers might improve the oral health status of people with dementia. Finally, periodontal disease might contribute to the onset or progression of dementia.
\end{abstract}

Keywords: oral health; aging population; oral disease; association with periodontal disease; oral care need; oral care strategies

\section{Introduction}

The transition from high to low mortality and fertility that accompanied the socioeconomic development of this century has meant a shift in the leading causes of disease and death and an increase of general health problems [1].

As a consequence, a decline of the oral health conditions of the elderly, such as dental caries and periodontal disease, is to be expected [2]. Poor oral health is more common in the elderly suffering from dementia, a disorder that will become prevalent with advancing age of the world population. Several studies analyzed the relationship between poor oral health and cognitive impairment, which suggests that cognitive decline might negatively impact oral health and also that poor oral health might lead to cognitive decline via specific biological mechanisms [3]. Conversely, in a recent systematic 
review by Wu et al. it has been argued that, according to some studies, it is unclear how or whether oral health conditions and cognitive status are related [4].

In cognitively impaired elderly, the increased incidence of oral disease might be favored by their general conditions: cognitive decline, loss of memory, learning disabilities, attention deficits, and motor skills deterioration, which result in reduced ability to perform routine oral care [5]. A frequent difficulty among these subjects is also the refusal of oral hygiene care, not opening their mouth, using abusive language, or being aggressive.

Even in the early stages of the disease progression, the elderly with dementia have a reduced salivary flow, which can lead not only to a higher prevalence of dental caries [6], but also to difficulties with eating and swallowing, which compromises communication skills [7]. The study by Silva et al. demonstrated that people with dementia living in Australians nursing homes have higher levels of untreated coronal and root caries, due to their cognitive condition and to poor access to professional services [8].

Periodontal disease is higher in the elderly with dementia than in normal cognitive individuals and the periodontal status worsens with the cognitive impairment progression [9]. Evidence of the association between periodontal disease and dementia has been demonstrated: periodontal disease is an inflammatory illness that affects the mouth and it could systemically affect individuals who are vulnerable to dementia and contribute to its pathogenesis [10].

Oral mucosal lesions are frequent in the elderly: the most common denture-related lesions are stomatitis, angular cheilitis, ulcers, hyperplasia, or candidosis. Reduced cognitive independence and decline in self-care, due to dementia, delirium, and social isolation, and it could make oral mucosal condition even worse [11].

Oral health problems in non-verbal individuals could negatively impact life quality, since they cannot communicate their pain and discomfort. On this topic, the study by Merlijn W. de Vries et al. proved the reliability of "the Orofacial Pain Scale for Non-Verbal Individuals" (OPS-NVI) and its psychometric evaluation (Delwel et al. 2018), to establish the presence of pain-related nonverbal communication, such as facial expressions, body movements, and vocal expressions [12].

\subsection{Rationale of the Systematic Review}

The most important risk factor that is associated with the onset of dementia is age and in an aging society, the impact of the phenomenon will be of alarming dimensions. Dementia reduces the quality of life of patients also with regard to oral health. It has been demonstrated that oral health of the elderly with dementia is poorer than oral health in people with normal cognitive function [13,14]. However, it is currently unclear how or whether oral health and cognitive status are related $[15,16]$. This study is aimed at reviewing literature, in order to evaluate oral health status in elderly with dementia, while comparing the data with those of elderly without dementia and to establish and assess the need of specific oral care strategies, which could improve their quality of life. This research was also conducted to review the available data regarding the influence of periodontal disease on the progression of cognitive impairment.

\subsection{Objectives}

The purpose of this systematic review was to examine studies about oral health in elderly with and without dementia, focusing the research on coronal and roots caries, number of remained teeth and retained roots, Decayed Missing Filled Index, periodontal disease, utilization of dentures, salivary flow, oral hygiene, oral mucosal lesions, orofacial pain, and on the analysis of periodontal disease as a potential risk factor for dementia.

This study reviewed cohort, case-control, cross-sectional studies, and randomized controlled clinical trials in order to examine oral health comparing the elderly with and without dementia. Inclusion criteria of participants were a diagnosis of dementia and the availability of data related to their oral health. 


\subsection{Clinical Question (PICO):}

- P: A population of participants with diagnosis of dementia aged 60 years or older

- I: Analysis of the oral health status and of the association between periodontal disease and dementia

- C: Comparison between oral health of elderly with and without dementia

- O: Prevalence of oral disease (affecting hard and soft tissues) in elderly with dementia compared to those without dementia, to define their need of oral care. Role of tooth loss due to periodontal disease in the onset/progression of dementia.

\section{Material and Methods}

\subsection{Protocol and Registration}

Methods and inclusion criteria were selected following the PRISMA statement [17], which offers a protocol with respect to the reference items that were included in this systematic review.

\subsection{Eligibility Criteria}

Inclusion and Exclusion Criteria

In this systematic review, all of the articles concerning oral health in the elderly with dementia meeting the following requirements were included:

- Participants must have been diagnosed with dementia

- Quantitative data about oral health problems

- Participants had to be available

- Participants had to be 60 years or older

- Cohort, case-control, cross-sectional studies, and clinical trial were considered

The exclusion criteria were as follows:

- $\quad$ Case report and reviews

- No quantitative data available

- Age of participants below 60 years

\subsubsection{Search}

An electronic research was conducted to identify relevant studies that have been published within 2019, but no restrictions were imposed with regard to language of the primary studies or methodology. The following electronic databases were used: PubMed, CINAHL, and Cochrane Library. The keywords used were the same for all three databases and they were combined with the Boolean term "AND": "oral health", "aging population", "oral disease", "association with periodontal disease" and "OR": "oral care need", and "oral care strategies". The research was completed on May 2019.

\subsubsection{Study Selection}

Two researchers (G.M., D.L.) independently analyzed the title, abstract, and full text of each English article to identify those that were eligible for the systematic review, according to the inclusion and exclusion criteria established above. Disagreements between reviewers were resolved by consensus. Articles that were published in other languages were assessed by a native speaker specialized in medical language. Articles in which the diagnosis of dementia was not defined or quantitative data were not available were excluded.

\subsubsection{Data Collection Process}

Two reviewers (G.M., D.L.) extracted the data, who also checked their methodological and clinical heterogeneity (D.L.). The information extracted from each article were as follows: study design (cohort, 
case-control, cross-sectional studies, or randomized clinical trial), participants characteristics, such as age and diagnosis of dementia, and quantitative data on participants (outcome measures), including the number of teeth present, number of retained roots, DMFT Index (Decayed Missing Filled Teeth Index), edentulousness and dentures, coronal and root caries, periodontal health, and its association with the onset or progression of dementia, oral mucosal disease, salivary flow, orofacial pain, oral hygiene, and need for dental treatment. Means and percentages were used for the principal outcome measures.

\subsubsection{Quality assessment}

Newcastle-Ottawa scale (NOS) [18] was used to assess the quality of the studies (Tables 1-4). The highest score was 9 and the lowest one was 1 (average score was 5.5 or cohort studies, 6.1 for case-control studies, 4.6 for cross-sectional studies, and 5.5 for RCT). Most articles used the standardized method to examine oral health and the examination was considered to be adequate if a dentist performed it. With regard to "Comparability", 30 of all the studies controlled for age or gender or both and in the case-control, cross-sectional studies, and RCT only 5 (1.98\% of the non-cohort studies) described the non-response rate $[14,19,20]$. In almost all studies, the duration of follow-up period was longer than three months. The evaluated quality parameters are shown in Additional file 1-4.

Table 1. Quality assessment for cohort studies (Additional file 1).

\begin{tabular}{|c|c|c|c|c|c|c|c|}
\hline Cohort Studies & $\begin{array}{l}\text { Representativeness } \\
\text { of Exposed Cohort }\end{array}$ & $\begin{array}{c}\text { Selection of } \\
\text { Non Exposed } \\
\text { Cohort }\end{array}$ & $\begin{array}{l}\text { Ascertainment } \\
\text { of Exposure }\end{array}$ & $\begin{array}{l}\text { Demonstration } \\
\text { Outcome Not } \\
\text { Present at Start } \\
\text { of Study }\end{array}$ & Comparability & Outcome & Total \\
\hline $\begin{array}{c}\text { Chalmers et al. } \\
2002[21]\end{array}$ & + & - & - & - & ++ & ++- & 5 \\
\hline $\begin{array}{l}\text { Chalmers et al. } \\
2003 \text { [13] }\end{array}$ & + & - & - & + & ++ & +++ & 7 \\
\hline $\begin{array}{l}\text { Chalmers et al. } \\
2004 \text { [22] }\end{array}$ & + & - & - & - & - & ++- & 3 \\
\hline $\begin{array}{c}\text { Chen et al. } 2010 \\
\text { [23] }\end{array}$ & + & + & + & - & - & +++ & 5 \\
\hline $\begin{array}{l}\text { De Souza Rolim } \\
\text { et al. } 2014 \text { [24] }\end{array}$ & + & - & + & - & - & +-- & 3 \\
\hline $\begin{array}{l}\text { Dintica et al. } \\
2018 \text { [25] }\end{array}$ & + & - & + & + & ++ & +++ & 8 \\
\hline $\begin{array}{c}\text { Ellefsen et al. } \\
2009 \text { [26] }\end{array}$ & + & + & + & - & ++ & ++- & 7 \\
\hline $\begin{array}{l}\text { Hatipoglu et al. } \\
2011 \text { [27] }\end{array}$ & - & - & - & + & - & ++ & 3 \\
\hline $\begin{array}{l}\text { Hoben et al. } \\
2016 \text { [28] }\end{array}$ & + & - & + & - & +- & ++ & 5 \\
\hline $\begin{array}{c}\text { Jones et al. } 1993 \\
\text { [29] }\end{array}$ & + & - & - & + & ++ & ++- & 6 \\
\hline $\begin{array}{c}\text { Kato et al. } 2019 \\
\text { [30] }\end{array}$ & + & - & + & + & +- & -++ & 6 \\
\hline $\begin{array}{c}\text { Ide et al. } 2016 \\
\text { [31] }\end{array}$ & - & - & + & + & +- & +++ & 6 \\
\hline $\begin{array}{c}\text { Lee et al. } 2017 \\
{[10]}\end{array}$ & + & + & + & - & ++ & +++ & 8 \\
\hline Ship and & & & & & & & \\
\hline $\begin{array}{c}\text { Puckett } 1994 \\
\text { [32] }\end{array}$ & + & + & + & - & +- & ++- & 6 \\
\hline $\begin{array}{c}\text { Sumi et al. } 2012 \\
\text { [33] }\end{array}$ & + & - & + & - & - & ++ & 4 \\
\hline $\begin{array}{l}\text { Takeuchi et al. } \\
2017 \text { [34] }\end{array}$ & + & - & + & - & ++ & +++ & 7 \\
\hline $\begin{array}{c}\text { Yoo et al. } 2019 \\
\text { [35] }\end{array}$ & + & - & + & + & ++ & ++- & 7 \\
\hline $\begin{array}{l}\text { Zenthofer et al. } \\
2014 \text { [36] }\end{array}$ & - & - & + & + & - & +++ & 5 \\
\hline $\begin{array}{l}\text { Zenthofer et al. } \\
\quad 2016 \text { [37] }\end{array}$ & + & + & + & + & ++ & +++ & 5 \\
\hline
\end{tabular}

$+=$ star assigned; $-=$ star not assigned. 
Table 2. Quality assessment for case-control studies (Additional file 2).

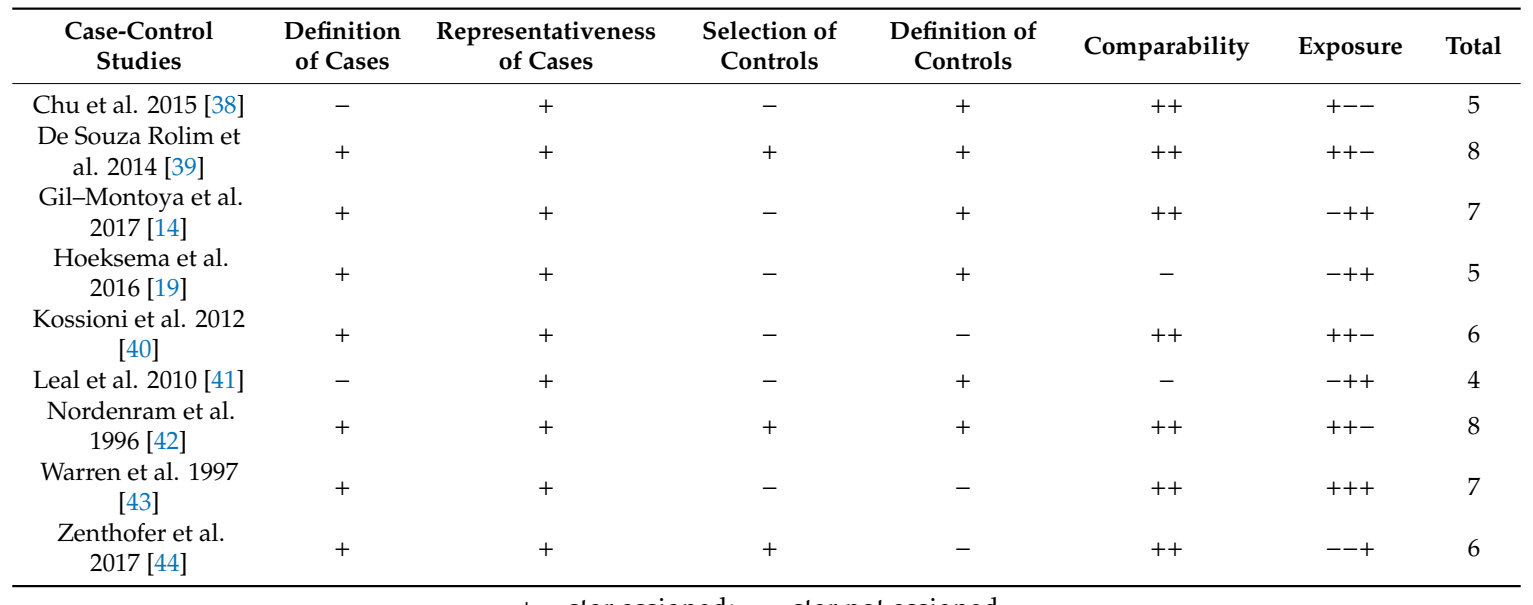

Table 3. Quality assessment for cross-sectional studies (Additional file 3).

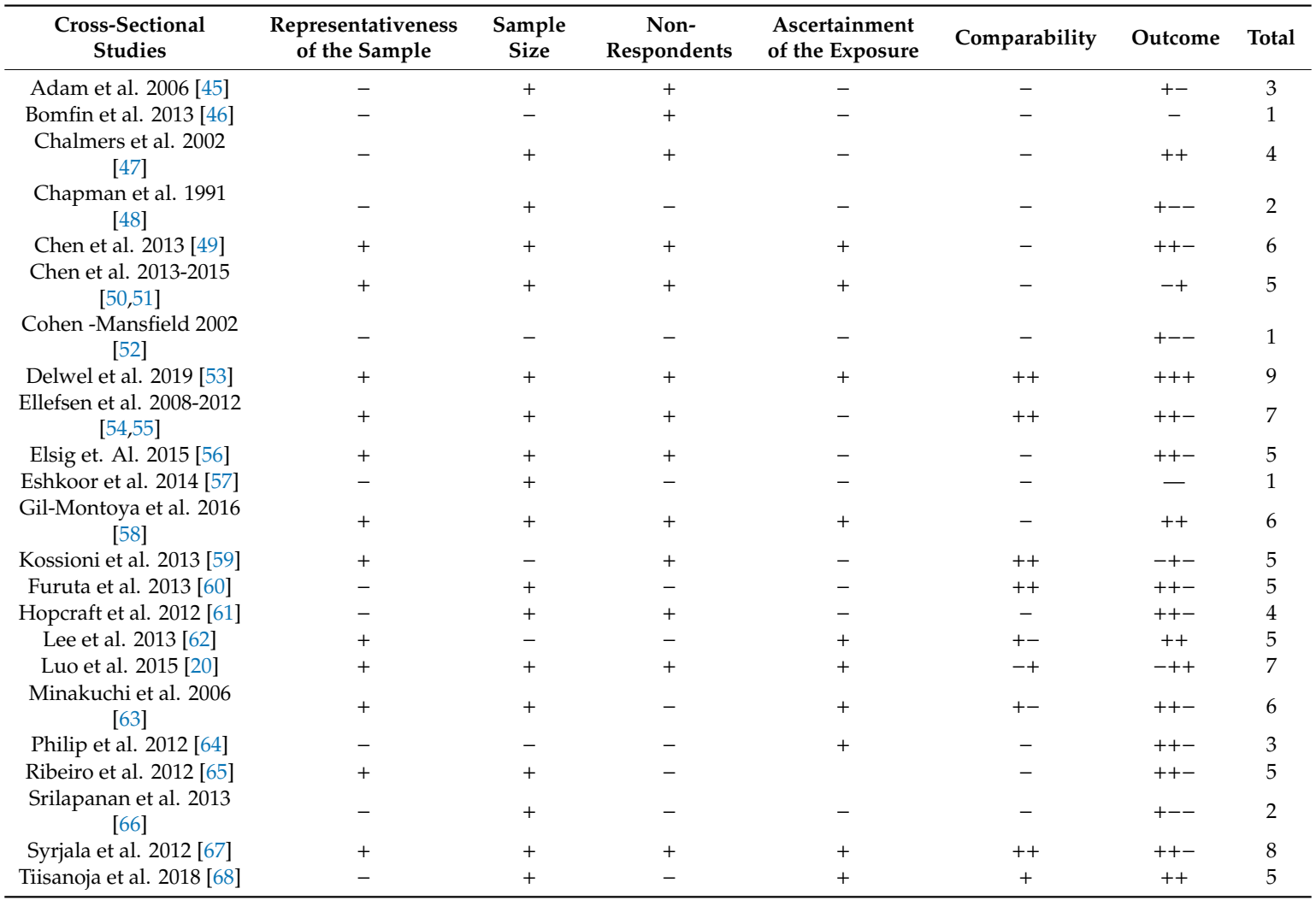

$+=$ star assigned $;-=$ star not assigned.

Table 4. Quality assessment for RCT (Additional file 4).

\begin{tabular}{|c|c|c|c|c|c|c|c|}
\hline $\begin{array}{c}\text { Randomized Clinical } \\
\text { Trial }\end{array}$ & Definition & $\begin{array}{c}\text { Representativeness } \\
\text { of Cases }\end{array}$ & $\begin{array}{l}\text { Selection of } \\
\text { Controls }\end{array}$ & $\begin{array}{l}\text { Definition of } \\
\text { Controls }\end{array}$ & Comparability & Exposure & Total \\
\hline Fjeld et al. 2014 [69] & + & - & + & + & & +++ & 6 \\
\hline Zenthofer et al. 2016 [44] & + & + & - & - & ++ & +-- & 5 \\
\hline
\end{tabular}




\section{Results}

\subsection{Study Selection and Characteristics}

A total of 922 studies that were published between 1990 and 2019 were identified from database searches. Among these articles, after examining titles and abstracts and the full texts of the remaining, only 56 met the inclusion criteria and were consequently included for quality assessment and data extraction. One study was added after scanning the reference list of the included articles [39]. All of the studies were analyzed with regard to quality while using the Newcastle-Ottawa Scale (NOS). Figure 1 shows the flow chart of publication assessment.

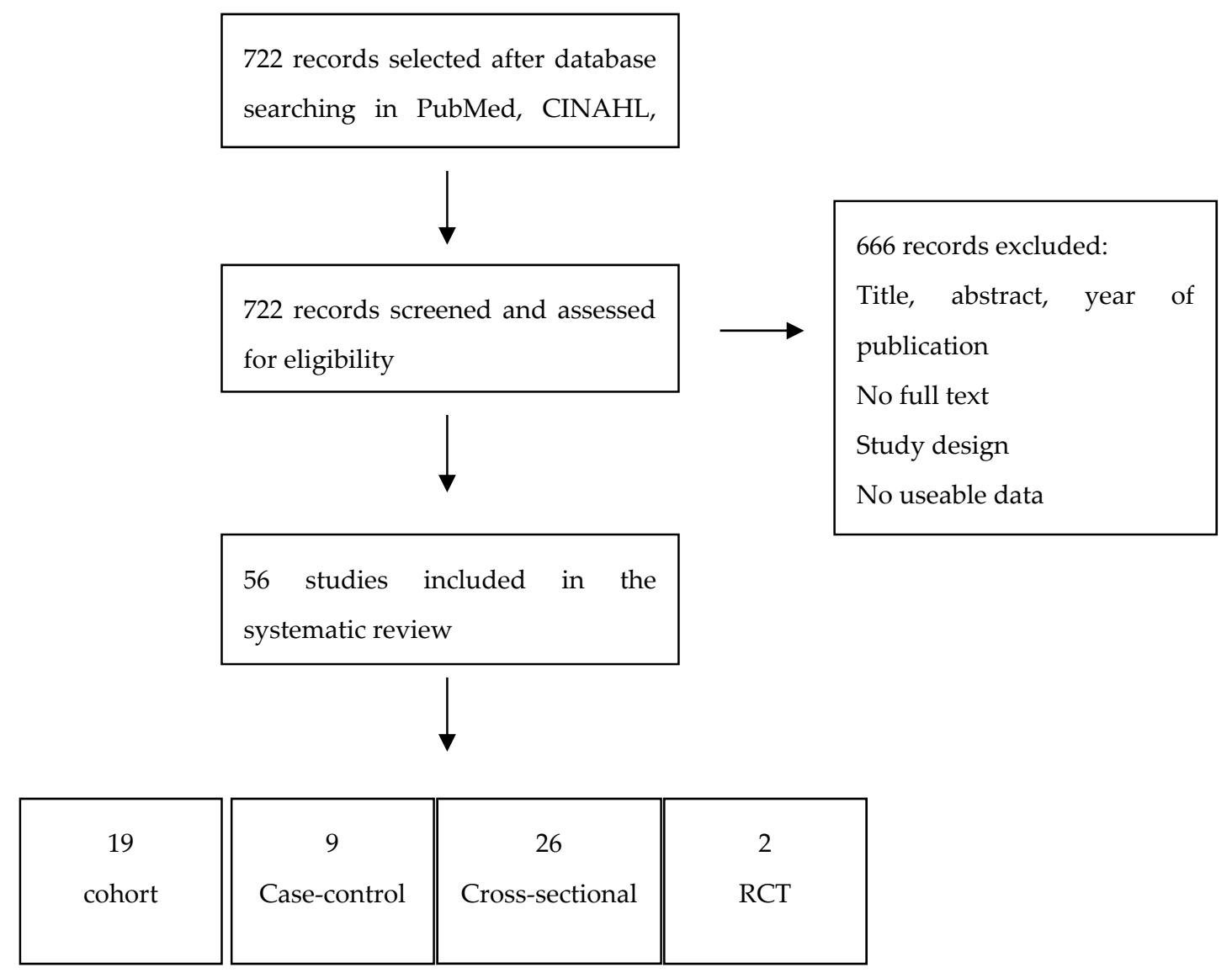

Figure 1. PRISMA flow diagram.

The detailed characteristics about the 56 included studies are presented in Table 5, and Table 6, with reference to author and year of publication, study design, case and controls, mean age of participants, dementia measure, and measure of oral health. This review included 19 cohort studies, 9 case-control-studies, 26 cross-sectional studies, and two randomized clinical trials. Almost all of the articles were in English, except for Sumi et al. article, which was written in Japanese [33]. A native Japanese speaker, who extracted the data included in the review, examined this article.

The studies that were selected for the review included in total 8466 participants with dementia and 6797 participants without dementia. In particular, selected studies regarding the association between periodontal disease and dementia included 4698 participants with periodontal disease or history of teeth extraction, 3132 elderly without periodontal disease, 60 subjects with dementia, and 2885 without dementia. In the included studies, the diagnosis of dementia was performed while using DSM-III and IV [70], ICD-9 and 10 (International Classification of Disease [71]), NINCDS-ADRDA (National Institute of Neurological and Communicative Disorders and Stroke and the Alzheimer's disease and 
Related Disorders Association [72]), Minimental State examination [73] (MMSE), and other additional measures (e.g., computed tomography, magnetic resonance imaging, CDR [74]).

Table 5. List of studies about coronal/root caries, number of remained teeth/retained roots, DMFT, periodontal disease, utilization of dentures, salivary flow, oral hygiene, and oral mucosal lesions in elderly with and without dementia.

\begin{tabular}{|c|c|c|c|c|c|c|}
\hline Study & Design & $\begin{array}{l}\text { Dementia } \\
\text { Group }\end{array}$ & Mean Age & $\begin{array}{l}\text { Control } \\
\text { Group }\end{array}$ & Mean Age & $\begin{array}{l}\text { Dementia and Oral } \\
\text { Health Measure }\end{array}$ \\
\hline $\begin{array}{c}\text { Adam et al. } 2006 \\
{[45]}\end{array}$ & Cross-sectional & 81 & 80.8 & 54 & 85.5 & $\begin{array}{c}\text { Abbreviated Mental } \\
\text { Test. } \\
\text { Orofacial pain, } \\
\text { dentures, } \\
\text { edentulousness, } \\
\text { DMFT, Debris Index }\end{array}$ \\
\hline $\begin{array}{l}\text { Chalmers et al. } \\
\text { 2002 [21] }\end{array}$ & Cohort & 116 & $\begin{array}{l}\text { 91: }<79 \text { years } \\
25: 80+\text { years }\end{array}$ & 116 & $\begin{array}{l}\text { 91: }<79 \text { years } \\
\text { 25: } 80+\text { years }\end{array}$ & $\begin{array}{c}\text { MMSE. } \\
\text { Number of present } \\
\text { teeth, DMFT, root } \\
\text { caries, Plaque Index }\end{array}$ \\
\hline $\begin{array}{l}\text { Chalmers et al. } \\
2003 \text { [13] }\end{array}$ & Cohort & 103 & $\begin{array}{l}82:<79 \text { years } \\
21: 80+\text { years }\end{array}$ & 113 & $\begin{array}{l}88:<79 \text { years } \\
25: 80+\text { years }\end{array}$ & $\begin{array}{c}\text { MMSE. } \\
\text { Number of present } \\
\text { teeth, DMFT, coronal } \\
\text { and root caries, } \\
\text { Plaque Index }\end{array}$ \\
\hline $\begin{array}{l}\text { Chalmers et al. } \\
\text { 2004 [22] }\end{array}$ & Cohort & 224 & 83.2 & 0 & - & $\begin{array}{c}\text { MMSE. } \\
\text { Orofacial pain, } \\
\text { number of present } \\
\text { teeth, DMFT, dental } \\
\text { habits, number of } \\
\text { coronal and root } \\
\text { caries, retained roots }\end{array}$ \\
\hline $\begin{array}{c}\text { Chen et al. } 2010 \\
\text { [23] }\end{array}$ & Cohort & 119 & 81.5 & 372 & 73.8 & $\begin{array}{c}\text { Chart. } \\
\text { Present of calculus, } \\
\text { plaque and gingival } \\
\text { bleeding }\end{array}$ \\
\hline $\begin{array}{c}\text { Chen et al. } 2013 \\
\text { [49] }\end{array}$ & Cross-sectional & $\begin{array}{c}\text { Community: } 51 \\
\text { Assisted living: } \\
18 \\
\text { NHR: } 501\end{array}$ & 79.3 & 0 & - & $\begin{array}{c}\text { Chart, ICD-9. } \\
\text { Number of present } \\
\text { teeth, decay or } \\
\text { retained roots, } \\
\text { presence of calculus, } \\
\text { plaque and gingival } \\
\text { bleeding, need for } \\
\text { oral care }\end{array}$ \\
\hline $\begin{array}{c}\text { Chen et al. } 2013 \\
\text { [50] }\end{array}$ & Cross-sectional & 501 & 82.6 & 199 & 76.1 & $\begin{array}{c}\text { Chart. } \\
\text { Presence of calculus, } \\
\text { plaque and gingival } \\
\text { bleeding }\end{array}$ \\
\hline $\begin{array}{l}\text { Chen et al. } 2015 \\
\text { [51] }\end{array}$ & Cross-sectional & 46 & 79.3 & 138 & 71.6 & $\begin{array}{c}\text { Chart. } \\
\text { Need for oral care }\end{array}$ \\
\hline $\begin{array}{l}\text { Chu et al. } 2015 \\
\text { [38] }\end{array}$ & Case-control & 59 & 79.8 & 59 & 79.8 & $\begin{array}{c}\text { Chart. } \\
\text { DMFT, Community } \\
\text { Periodontal Index, } \\
\text { Salivary flow }\end{array}$ \\
\hline
\end{tabular}


Table 5. Cont.

\begin{tabular}{|c|c|c|c|c|c|c|}
\hline Study & Design & $\begin{array}{l}\text { Dementia } \\
\text { Group }\end{array}$ & Mean Age & $\begin{array}{l}\text { Control } \\
\text { Group }\end{array}$ & Mean Age & $\begin{array}{l}\text { Dementia and Oral } \\
\text { Health Measure }\end{array}$ \\
\hline $\begin{array}{l}\text { Cohen-Mansfield } \\
\text { 2002 [52] }\end{array}$ & Cross-sectional & 21 & 88.0 & 0 & - & $\begin{array}{l}\text { MMSE, MDS-COGS. } \\
\text { Number of broken or } \\
\text { fractured teeth, caries, } \\
\text { retained roots, } \\
\text { dentures, presence of } \\
\text { gingivitis and } \\
\text { periodontal disease }\end{array}$ \\
\hline $\begin{array}{l}\text { De Souza Rolim } \\
\text { et al. } 2014 \text { [24] }\end{array}$ & Case-control & 29 & 75.2 & 30 & 61.2 & $\begin{array}{l}\text { NINCDS-ADRDA for } \\
\text { AD, MMSE. } \\
\text { Orofacial pain, DMFT, } \\
\text { Gingival Bleeding } \\
\text { Index, probing pocket } \\
\text { depth, CAL, Plaque } \\
\text { Index }\end{array}$ \\
\hline $\begin{array}{l}\text { Delwel et al. } \\
2019 \text { [53] }\end{array}$ & Cross-sectional & $\begin{array}{l}\text { Dementia:303 } \\
\text { MCI: } 45\end{array}$ & & - & - & $\begin{array}{c}\text { MMS. } \\
\text { Presence of orofacial } \\
\text { pain using the } \\
\text { OPS-NVI and } \\
\text { self-reported pain, } \\
\text { number of missing or } \\
\text { restored teeth, coronal } \\
\text { and root caries, } \\
\text { retained roots, DPSI, } \\
\text { Plaque Index of } \\
\text { Silness and Loë, oral } \\
\text { hygiene, oral mucosal } \\
\text { lesions }\end{array}$ \\
\hline $\begin{array}{l}\text { Ellefsen et al. } \\
2009 \text { [26] }\end{array}$ & Cohort & $\begin{array}{l}\text { AD: } 49 \\
\text { OD:15 }\end{array}$ & $\begin{array}{l}83.6 \\
81.3\end{array}$ & 13 & 79.9 & $\begin{array}{c}\text { ICD-10. } \\
\text { Number of present } \\
\text { teeth, DMFT, CCI, } \\
\text { NCI, ADJCI }\end{array}$ \\
\hline $\begin{array}{l}\text { Ellefsen et al. } \\
2012 \text { [55] }\end{array}$ & Cross-sectional & 61 & 82.8 & 0 & - & $\begin{array}{l}\text { Number of present } \\
\text { teeth, DMFT, coronal } \\
\text { caries, root caries }\end{array}$ \\
\hline $\begin{array}{l}\text { Elsig et al. } 2013 \\
\text { [56] }\end{array}$ & Cross-sectional & 29 & 82.5 & 22 & 81.9 & $\begin{array}{c}\text { NPT, MMSE, CERAD, } \\
\text { CDR. } \\
\text { Number of present } \\
\text { teeth, presence of } \\
\text { visible dental plaque }\end{array}$ \\
\hline $\begin{array}{l}\text { Eshkoor et al. } \\
\text { 2014 [57] }\end{array}$ & Cross-sectional & 1210 & 71.0 & 0 & - & $\begin{array}{c}\text { MMSE. } \\
\text { Presence of teeth or } \\
\text { dentures }\end{array}$ \\
\hline $\begin{array}{l}\text { Fjeld et al. } 2014 \\
\text { [69] }\end{array}$ & $\mathrm{RCT}$ & 159 & 85.5 & 43 & 88.5 & $\begin{array}{c}\text { Evaluated by } \\
\text { physician. } \\
\text { Number of present } \\
\text { teeth, Simplified Oral } \\
\text { Hygiene Index, } \\
\text { mouth dryness }\end{array}$ \\
\hline
\end{tabular}


Table 5. Cont

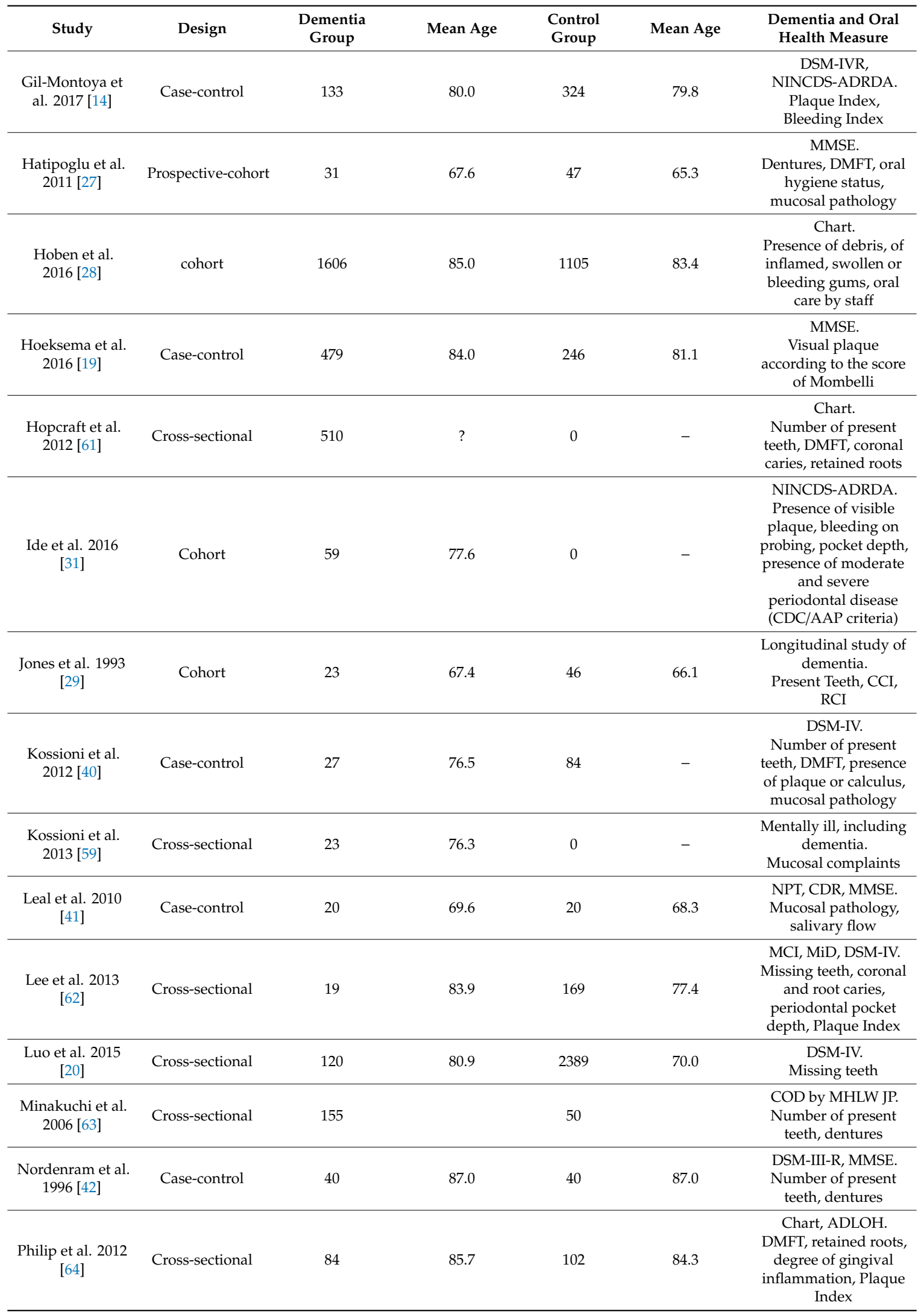


Table 5. Cont.

\begin{tabular}{|c|c|c|c|c|c|c|}
\hline Study & Design & $\begin{array}{l}\text { Dementia } \\
\text { Group }\end{array}$ & Mean Age & $\begin{array}{l}\text { Control } \\
\text { Group }\end{array}$ & Mean Age & $\begin{array}{l}\text { Dementia and Oral } \\
\text { Health Measure }\end{array}$ \\
\hline $\begin{array}{l}\text { Ribeiro et al. } \\
2012 \text { [65] }\end{array}$ & Cross-sectional & 30 & 79.1 & 30 & 67.8 & $\begin{array}{c}\text { ICD-10, DSM-IV, } \\
\text { MMSE, CDR. } \\
\text { Number of present } \\
\text { teeth, dentures, } \\
\text { DMFT, Oral Health } \\
\text { Index }\end{array}$ \\
\hline $\begin{array}{l}\text { Sumi et al. } 2012 \\
\text { [33] }\end{array}$ & Cohort & 10 & 77.7 & 0 & - & $\begin{array}{l}\text { NINCD-ADRDA, } \\
\text { MMSE. } \\
\text { Number of present } \\
\text { teeth, DMFT, Gingival } \\
\text { Index, Plaque Index }\end{array}$ \\
\hline $\begin{array}{l}\text { Syrjala et al. } \\
2012 \text { [67] }\end{array}$ & Cross-sectional & 49 & 84.8 & 278 & 81.4 & $\begin{array}{l}\text { DSM-III-R, DSM-IV, } \\
\text { McKeith. } \\
\text { Number of present } \\
\text { teeth, dentures, } \\
\text { number of teeth with } \\
\text { periodontal pockets }> \\
4 \text { mm, presence of } \\
\text { poor oral hygiene }\end{array}$ \\
\hline $\begin{array}{l}\text { Zenthöfer et al. } \\
2014 \text { [36] }\end{array}$ & Cohort & 57 & 83.1 & 36 & 82.6 & $\begin{array}{c}\text { MMSE, chart. } \\
\text { Decayed and missing } \\
\text { teeth, periodontitis, } \\
\text { Gingival Bleeding } \\
\text { Index, CPITN, Dental } \\
\text { Hygiene Index, } \\
\text { Plaque Control } \\
\text { Record }\end{array}$ \\
\hline $\begin{array}{l}\text { Zenthöfer et al. } \\
\text { 2016 [37] }\end{array}$ & Cohort & 33 & 81.7 & 60 & 83.4 & $\begin{array}{c}\text { MMSE, chart. } \\
\text { Missing teeth, } \\
\text { Gingival Bleeding } \\
\text { Index, CPITN, Plaque } \\
\text { Control Record, } \\
\text { mucosa pathology }\end{array}$ \\
\hline
\end{tabular}


Table 5. Cont.

\begin{tabular}{|c|c|c|c|c|c|c|}
\hline Study & Design & $\begin{array}{l}\text { Dementia } \\
\text { Group }\end{array}$ & Mean Age & $\begin{array}{l}\text { Control } \\
\text { Group }\end{array}$ & Mean Age & $\begin{array}{c}\text { Dementia and Oral } \\
\text { Health Measure }\end{array}$ \\
\hline $\begin{array}{l}\text { Zenthöfer et al. } \\
2017 \text { [44] }\end{array}$ & Case-control & 136 & 84.6 & 83 & 80.7 & $\begin{array}{c}\text { MMSE. } \\
\text { Gingival Bleeding } \\
\text { Index, CPITN }\end{array}$ \\
\hline \multicolumn{7}{|c|}{$\begin{array}{l}\text { ADJCI: adjusted caries increment; CAL: clinical attachment level; CCI: crude caries increment; CDC/AAP: Center } \\
\text { for Disease Control/American Academy of Periodontology; COD: classification of dementia; CPITN: community } \\
\text { periodontal index of treatment needs CT: computer tomography; DMFT: decayed missing filled teeth; DPSI: Dutch } \\
\text { Periodontal Screening Index; ICD: International Classification of Disease; MCI: Mild Cognitive Impairment; Mckeith: } \\
\text { Consensus criteria presented by McKeith; MDS-COGS: Minimum Data Set Cognition Scale; MHLW: Ministry of } \\
\text { Health, Labour and Welfare; MMSE: Mini Mental State Examination; MRI: magnetic resonance imaging; NCI: net } \\
\text { caries increment NINCDS-ADRDA: National Institute of Neurological Disorders and Stroke Alzheimer's Disease and } \\
\text { Related Disorders Association; NHR: nursing home residents; NPT: Neuropsychological testing; NT: Neurological } \\
\text { testing; OPS-NVI: ORrofacial Pain Scale for Nonverbal Individuals; PET. Positron emission tomography. }\end{array}$} \\
\hline
\end{tabular}

Table 6. List of studies about the relationship between dementia and periodontal disease.

\begin{tabular}{|c|c|c|c|c|c|c|}
\hline Study & Design & $\begin{array}{c}\text { Dementia or } \\
\text { Periodontal } \\
\text { Disease Group }\end{array}$ & Mean Age & $\begin{array}{l}\text { Control } \\
\text { Group }\end{array}$ & Mean Age & $\begin{array}{c}\text { Dementia and Oral } \\
\text { Periodontal Disease } \\
\text { Measure: }\end{array}$ \\
\hline $\begin{array}{c}\text { Dintica et al. } \\
2018 \text { [25] }\end{array}$ & Cohort & Free D: 2715 & - & - & & $\begin{array}{l}\text { Dementia incidence: } \\
\text { DSM-IV, MMSE. }\end{array}$ \\
\hline $\begin{array}{l}\text { Ide et al. } 2016 \\
\text { [31] }\end{array}$ & Cohort & $\begin{array}{l}\text { Mid to Mod: } 60 \\
30 \text { men } \\
30 \text { women }\end{array}$ & 77.7 & - & - & $\begin{array}{c}\text { Presence or absence of } \\
\text { periodontal disease } \\
\text { following CDC/AAP }\end{array}$ \\
\hline $\begin{array}{l}\text { Takeuchi et al. } \\
2017 \text { [34] }\end{array}$ & Cohort & $\begin{array}{l}\text { PD: } 1566(691 \\
\text { men and } 875 \\
\text { women) }\end{array}$ & - & - & - & $\begin{array}{c}\text { Dementia incidence: } \\
\text { DSM-III, MMSE, } \\
\text { HDS-R. }\end{array}$ \\
\hline $\begin{array}{l}\text { Tiisanoja et al. } \\
2018 \text { [68] }\end{array}$ & Cross-sectional & IG: 170 & $\begin{array}{c}80.9 \\
\text { AD: } 82.2 \\
\text { OD: } 82.5\end{array}$ & - & - & $\begin{array}{l}\text { DSM-IV, McKeith et } \\
\text { al. MMSE. } \\
\text { Structured oral } \\
\text { examination } \\
\text { examining number of } \\
\text { teeth with periodontal } \\
\text { pocket depth of } 4 \mathrm{~mm} \\
\text { or more }\end{array}$ \\
\hline
\end{tabular}

\footnotetext{
AD: Alzheimer's disease; CDC/AAP: Centre for Disease Control/American Academy of Periodontology; FreeD: Free-dementia; DI: Dementia incidence; HDS-R: Revised Hasegawa's Dementia Scale; IG: Intervention Group; Mid to Mod: mild to moderate dementia; OD: other dementia; PD: Periodontal Disease; NHANES: National Health and Nutrition Examination Survey; NE: non-extraction cohort; TE: tooth extraction.
}

\subsection{Results of Individual Studies}

The number of present teeth was one of the most used measures for assessing oral health. In particular, from the included studies it came to light that the range within the two groups varied between 2.0 to 20 . 2 for people without dementia and between 1.7 to 20.5 for people with dementia. According to Delwel et al. [53], the number of present teeth was lower in the participants with dementia (median = 2.0, IQR = 0.0-18.0) than in people with MCI (median = 18.0, IQR = 5.5-24.0). However, this study underlined that, if only dentate participants were considered, no significant differences were recorded between the two groups (median $=18.0, \mathrm{IQR}=9.0-24-0)$ (Table 7). The review showed that 
coronal and root caries were more common in people with dementia that in people without dementia: coronal caries varied between $0.1-2.9[43,54,55]$ and $0.0-1-0$, respectively $[21,54]$, and root caries varied between $0.6-4.9[43,54,55]$ in participants with dementia and $0.3-1.7[13,54,55]$ in normal cognitive participants. For retained roots, the range was between 0.0-1.2 [54] in people without dementia and between 0.2-10 [21,54] in people with dementia. Moreover, Delwel et al. [53] demonstrated that dentate participants with dementia had more coronal caries (median $=1.0, \mathrm{IQR}=0.0-2.0$ ), root caries, and retained roots (median $=0.0, \mathrm{IQR}=0.0-1.0$ ) than dentate people with $\mathrm{MCI}$ (Table 8 . With regards to the DMFT Index (Table 9), the lowest one was 14.9 in the study by Srilapanan et al. In general, the DMFT index did not show a significant difference within the two groups (19.7-26.1 in healthy people and 14.9-28.0 in people with dementia), except for one study, which demonstrated that the DMFT Index was 25.5 in people without dementia and 28.0 in people with dementia.

Most of the participants with dementia presented gingival bleeding or inflammation $[23,48]$ (Table 10). According to De Souza [24], the Gingival Bleeding Index was 46.0\% in the elderly with dementia and periodontal infections were most common in the latter (58.6\%) than in normal cognitive participants (26.7\%). $73.8 \%$ of the Delwel et al. [53] study included patients had periodontal pockets of $\geq 4 \mathrm{~mm}, 18.8 \%$ of them had one or more teeth with mobility grade 2 , and $5.8 \%$ had one or more teeth with mobility grade 3 .

Table 7. Results about the number of present teeth.

\begin{tabular}{|c|c|c|c|}
\hline Study & $\begin{array}{l}\text { Number of Present } \\
\text { Teeth No Dementia }\end{array}$ & $\begin{array}{l}\text { Number of Present } \\
\text { Teeth Dementia }\end{array}$ & $\begin{array}{l}p \text { Value: Dementia } \\
\text { vs. No Dementia }\end{array}$ \\
\hline Bomfin et al. 2013 [46] & 2.0 & 3.0 & - \\
\hline $\begin{array}{c}\text { Chalmers et al. 2002, } 2003 \\
{[13,21,47]}\end{array}$ & 17.2 & 18.0 & $>0.05$ \\
\hline Chapman et al. 1991 [48] & - & 12.8 & - \\
\hline Chen et al. 2010 [23] & - & $\begin{array}{l}18.2 \text { community living } \\
19.3 \text { assisted living }\end{array}$ & - \\
\hline Delwel et al. 2019 [53] & - & $\begin{array}{l}\text { Dementia:18.0 } \\
\text { MCI: } 22.0\end{array}$ & 0.058 \\
\hline Ellefsen et al. 2009 [26] & 20.2 & $\begin{array}{l}17.3 \mathrm{AD} \\
16.1 \mathrm{OD}\end{array}$ & $\leq 0.001$ \\
\hline Ellefsen et al. 2012 [55] & - & 16.5 & - \\
\hline Elsig et al. 2013 [56] & 6.5 & 4.9 & 0.533 \\
\hline Fjeld et al. 2013 [69] & 20.1 & 20.0 & - \\
\hline Hopcraft et al. 2012 [61] & 14.6 & 0.7 & $>0.05$ \\
\hline Jones et al. 1993 [29] & 18.2 & 17.9 & 0.90 \\
\hline Kossioni et al. 2012 [40] & - & 4.4 & - \\
\hline Ribeiro et al. 2012 [65] & Median 13.5 (0.0-28-0) & Median 1.0 (o.o-22-0) & 0.0004 \\
\hline Srisilapanan et al. 2013 [66] & - & 19.5 & - \\
\hline Sumi et al. 2012 [33] & - & 12.7 & - \\
\hline Syrjala et al. 2012 [67] & 15.0 & $\begin{array}{c}10.9 \mathrm{AD} \\
7.8 \mathrm{VaD} \\
1.7 \mathrm{OD}\end{array}$ & - \\
\hline Warren et al. 1997 [43] & 13.0 & $\begin{array}{l}\text { 10.0 AD } \\
\text { 13.0 OD }\end{array}$ & $p>0.05$ \\
\hline
\end{tabular}

AD: Alzheimer dementia; OD: other dementia; Vad: vascular dementia. 
Table 8. Results about coronal/root caries and retained roots.

\begin{tabular}{|c|c|c|c|c|c|c|}
\hline Study & $\begin{array}{c}\text { Coronal } \\
\text { Caries No } \\
\text { Dementia }\end{array}$ & $\begin{array}{c}\text { Coronal } \\
\text { Caries } \\
\text { Dementia }\end{array}$ & $\begin{array}{c}\text { Root caries } \\
\text { no } \\
\text { Dementia }\end{array}$ & $\begin{array}{l}\text { Root Caries } \\
\text { Dementia }\end{array}$ & $\begin{array}{l}\text { Retained } \\
\text { Roots no } \\
\text { Dementia }\end{array}$ & $\begin{array}{l}\text { Retained Root } \\
\text { Dementia }\end{array}$ \\
\hline $\begin{array}{l}\text { Chalmers et al. } \\
\text { 2002-2003 [13,47] }\end{array}$ & $0.0^{*}$ & $* 0 * 5$ & 0.3 & 0.8 & $\begin{array}{l}\text { Decayed: } 0.0 * \\
\text { Sound: } 0.1\end{array}$ & $\begin{array}{l}\text { Decayed: } 0.3^{*} \\
\text { Sound: } 0.1\end{array}$ \\
\hline $\begin{array}{l}\text { De Souza Rolim et al. } \\
2014[24,39]\end{array}$ & $3.4 \%$ & $6.8 \%$ & - & - & $10.2 \%$ & $6.8 \%$ \\
\hline Delwel et al. 2019 [53] & - & $27.0 \%$ & - & $19.0 \%$ & - & $18.1 \%$ \\
\hline $\begin{array}{l}\text { Ellefsen et al. 2008-2012 } \\
{[54,55]}\end{array}$ & $1.0^{*}$ & $2.9 *$ & $1.7^{*}$ & $\begin{array}{l}4.9 * \mathrm{AD} \\
2.3 * \mathrm{OD}\end{array}$ & 0.0 & $\begin{array}{l}\text { AD } 10.0 * \\
\text { OD } 0.5^{*}\end{array}$ \\
\hline Jones et al. 1993 [29] & 0.8 & 1.4 & 0.4 & 1.8 & - & - \\
\hline Lee et al. 2013 [62] & 0.8 & 1.0 & $0.5^{*}$ & $1.8^{*}$ & - & - \\
\hline Philip et al. 2012 [64] & - & - & - & - & 1.2 & 1.8 \\
\hline Warren et al. 1997 [43] & 0.4 & $\begin{array}{l}\text { AD } 0.1 \\
\text { OD } 0.4\end{array}$ & 0.8 & $\begin{array}{l}\text { AD O.6 } \\
\text { OD } 0.6\end{array}$ & - & - \\
\hline
\end{tabular}

AD: Alzheimer's disease; OD: other dementia; $: p \leq 0.05 ;{ }^{* *}: p \leq 0.01$.

Table 9. Results about the DMFT Index.

\begin{tabular}{|c|c|c|}
\hline Study & DMFT No Dementia & DMFT Dementia \\
\hline Adam et al. 2006 [45] & $\begin{array}{l}\text { Decayed } 1.1 \\
\text { Missing } 28.2 \\
\text { Filled } 0.7\end{array}$ & $\begin{array}{l}\text { Decayed } 0.80 \\
\text { Missing } 27.3 \\
\text { Filled } 0.90\end{array}$ \\
\hline Chalmers et al. $2002[21,47]$ & $\begin{array}{l}\text { Decayed 0.0-0.4 } \\
\quad \text { Missing - } \\
\text { Filled 24.7-25.7 }\end{array}$ & $\begin{array}{l}\text { Decayed 0.5-1.6* } \\
\text { Missing - } \\
\text { Filled 22.1-23.9 }\end{array}$ \\
\hline Chalmers et al. 2003 [13] & $\begin{array}{l}\text { Decayed 0.0-0.1 } \\
\text { Missing - } \\
\text { Filled - }\end{array}$ & $\begin{array}{l}\text { Decayed } 0.3-1.3 * \\
\text { Missing - } \\
\text { Filled - }\end{array}$ \\
\hline Chapman et al. 1991 [48] & $\begin{array}{l}\text { Decayed - } \\
\text { Missing - } \\
\text { Filled - }\end{array}$ & $\begin{array}{c}\text { Decayed } 1.4 \\
\text { Missing } 17.8 \\
\text { Filled } 6.4 \\
\text { DMFT } 25.6\end{array}$ \\
\hline Chen et al. $2013[49,50]$ & $\begin{array}{l}\text { Decayed - } \\
\text { Missing - } \\
\text { Filled - }\end{array}$ & $\begin{array}{c}\text { Decayed } 5.5(\mathrm{C}), 5.3(\mathrm{~A}), 6.0(\mathrm{NHR}) \\
\text { Missing - } \\
\text { Filled } 10.4(\mathrm{C}), 10.9(\mathrm{~A}), 8.7(\mathrm{NHR})\end{array}$ \\
\hline Chu et al. 2015 [38] & $\begin{array}{l}\text { Decayed } 0.8 \\
\text { Missing } 18.3 \\
\text { Filled } 2.4 \\
\text { DMFT } 21.5\end{array}$ & $\begin{array}{l}\text { Decayed } 1.2 \\
\text { Missing } 18.9 \\
\text { Filled } 2.5 \\
\text { DMFT } 22.3\end{array}$ \\
\hline De Souza Rolim et al. 2014 [24,39] & $\begin{array}{l}\text { Decayed - } \\
\text { Missing - } \\
\text { Filled - }\end{array}$ & $\begin{array}{c}\text { Decayed - } \\
\text { Missing - } \\
\text { Filled - } \\
\text { DMFT 27.2 Range 11-32 }\end{array}$ \\
\hline Hatipoglu et al. 2011 [27] & $\begin{array}{l}\text { Decayed } \\
\text { Missing } \\
\text { Filled } \\
\text { DMFT } 19.7\end{array}$ & $\begin{array}{l}\text { Decayed } \\
\text { Missing } \\
\text { Filled } \\
\text { DMFT } 24.2\end{array}$ \\
\hline Hopcraft et al. 2012 [61] & $\begin{array}{l}\text { Decayed } 2.9 \\
\text { Missing } 17.4 \\
\text { Filled } 4.8 \\
\text { DMFT } 25.0\end{array}$ & $\begin{array}{c}\text { Decayed } 2.4 \\
\text { Missing } 17.9 \\
\text { Filled } 4.8 \\
\text { DMFT } 25.0\end{array}$ \\
\hline
\end{tabular}


Table 9. Cont.

\begin{tabular}{|c|c|c|}
\hline Study & DMFT No Dementia & DMFT Dementia \\
\hline Kossioni et al. 2012 [40] & $\begin{array}{l}\text { Decayed - } \\
\text { Missing - } \\
\text { Filled - }\end{array}$ & $\begin{array}{c}\text { Decayed } 1.8 \\
\text { Missing - } \\
\text { Filled 0.9 }\end{array}$ \\
\hline Lee et al. 2013 [62] & $\begin{array}{c}\text { Decayed CC+RC } \\
\text { Missing } 12.7 \\
\text { Filled - }\end{array}$ & $\begin{array}{c}\text { Decayed CC }+\mathrm{RC} \\
\text { Missing } 10.2 \\
\text { Filled - }\end{array}$ \\
\hline Luo et al. 2015 [20] & $\begin{array}{l}\text { Decayed - } \\
\text { Missing } 9.3 \\
\text { Filled - }\end{array}$ & $\begin{array}{l}\text { Decayed - } \\
\text { Missing } 18.7^{* *} \\
\text { Filled - }\end{array}$ \\
\hline Philip et al. 2012 [64] & $\begin{array}{c}\text { Decayed } 2.9 \\
\text { Missing } 18.0 \\
\text { Filled 5.0 } \\
\text { DMFT } 26.1\end{array}$ & $\begin{array}{c}\text { Decayed } 3.0 \\
\text { Missing } 17.4 \\
\text { Filled } 5.3 \\
\text { DMFT } 25.9\end{array}$ \\
\hline Ribeiro et al. 2012 [65] & $\begin{array}{l}\text { Decayed } \\
\text { Missing } \\
\text { Filled } \\
\text { DMFT 25.5 * }\end{array}$ & $\begin{array}{c}\text { Decayed } \\
\text { Missing } \\
\text { Filled } \\
\text { DMFT 28.0* }\end{array}$ \\
\hline Srisilapanan et al. 2013 [66] & $\begin{array}{l}\text { Decayed - } \\
\text { Missing - } \\
\text { Filled - }\end{array}$ & $\begin{array}{c}\text { Decayed } 1.5 \\
\text { Missing } 12.6 \\
\text { Filled } 0.8 \\
\text { DMFT } 14.9\end{array}$ \\
\hline Zenthöfer et al. 2014 [36] & $\begin{array}{l}\text { Decayed } 0.7 \\
\text { Missing } 19.9 \\
\text { Filled - }\end{array}$ & $\begin{array}{l}\text { Decayed } 0.6 \\
\text { Missing } 20.8 \\
\text { Filled - }\end{array}$ \\
\hline Zenthöfer et al. 2016 [37] & $\begin{array}{l}\text { Decayed - } \\
\text { Missing } 20.5 \\
\text { Filled - }\end{array}$ & $\begin{array}{c}\text { Decayed - } \\
\text { Missing } 20.5 \\
\text { Filled - }\end{array}$ \\
\hline
\end{tabular}

CC: coronal caries; DMFT: decayed missing filled teeth; NHR: nursing homes residents; RC: root caries; ${ }^{*}: p \leq 0.05$; ${ }^{* *}: p \leq 0.01$.

Table 10. Results about gingival and periodontal disease.

\begin{tabular}{|c|c|c|c|}
\hline Study & Outcome Measure & $\begin{array}{l}\text { Gingival Health No } \\
\text { Dementia }\end{array}$ & $\begin{array}{l}\text { Gingival Health } \\
\text { Dementia }\end{array}$ \\
\hline Chen et al. 2010 [23] & $\begin{array}{l}\text { Calculus/plaque/gingival } \\
\text { bleeding }\end{array}$ & $\begin{array}{c}\text { No: } 1.2 \% \\
\text { M to M: } 85.5 \% \\
\text { High: } 13.3 \%\end{array}$ & $\begin{array}{c}\text { No: } 0.9 \% \\
\text { M to M: } 67.9 \% \\
\text { High: } 31.3 \%\end{array}$ \\
\hline Chen et al. 2013 [49] & $\begin{array}{l}\text { Calculus/plaque/gingival } \\
\text { bleeding }\end{array}$ & - & $\begin{array}{c}\text { C: } 0 \% \text { No, } 65.8 \% \text { Small, } \\
\text { 34.2 High } \\
\text { A: } 8.3 \% \text { No, } 66.7 \% \text { Small, } \\
25.0 \% \text { High } \\
\text { NHR: } 59.2 \% \text { No, } 59.2 \% \\
\text { Small, } 40.5 \% \text { High } \\
\end{array}$ \\
\hline Chen et al. 2013 [50] & $\begin{array}{l}\text { Calculus/plaque/gingival } \\
\text { bleeding }\end{array}$ & $\begin{array}{c}\text { No: } 0.0 \% \\
\text { M to M: } 73.8 \% \\
\text { High: } 26.2 \%\end{array}$ & $\begin{array}{c}\text { No: } 0.3 \% \\
\text { M to M: } 59.2 \% \\
\text { High: } 40.4 \%\end{array}$ \\
\hline Chu et al. 2015 [38] & $\begin{array}{l}\text { Community Periodontal } \\
\text { Index, pockets } \geq 3 \mathrm{~mm}\end{array}$ & $74.0 \%$ & $78.0 \%$ \\
\hline $\begin{array}{l}\text { Cohen-Mansfield et al. } \\
2002 \text { [52] }\end{array}$ & $\begin{array}{l}\text { Periodontal disease } \\
\text { Gingivitis }\end{array}$ & - & $\begin{array}{l}44.4 \% \\
38.9 \%\end{array}$ \\
\hline
\end{tabular}


Table 10. Cont.

\begin{tabular}{|c|c|c|c|}
\hline Study & Outcome Measure & $\begin{array}{c}\text { Gingival Health No } \\
\text { Dementia }\end{array}$ & $\begin{array}{l}\text { Gingival Health } \\
\text { Dementia }\end{array}$ \\
\hline $\begin{array}{l}\text { De Souza Rolim et al. } \\
2014 \text { [24] }\end{array}$ & $\begin{array}{c}\text { Probing pocket depth } \\
\text { (mm) } \\
\text { Gingivitis } \\
\text { MoD-SeV periodontitis } \\
\text { Periodontal infection } \\
\text { Gingival Bleeding Index }\end{array}$ & $\begin{array}{c}- \\
10.0 \%-10.0 \% \\
6.7 \% \\
26.7 \% \\
-\end{array}$ & $\begin{array}{c}1.6 \mathrm{~mm} \\
31.0 \% \\
6.9 \%-20.7 \% \\
58.6^{* *} \\
46.0 \%\end{array}$ \\
\hline $\begin{array}{l}\text { De Souza Rolim et al. } \\
2014 \text { [39] }\end{array}$ & $\begin{array}{c}\text { Probing pocket depth } \\
\text { (mm) } \\
\text { Gingivitis } \\
\text { MoD-SeV periodontitis } \\
\text { Periodontal infection } \\
\text { Gingival Bleeding Index }\end{array}$ & $\begin{array}{c}- \\
10.0 \%-10.0 \% \\
6.7 \% \\
26.7 \% \\
-\end{array}$ & $\begin{array}{c}1.6 \mathrm{~mm} \\
31.0 \% \\
6.9 \%-20.7 \% \\
58.6^{* *} \\
46.0 \%\end{array}$ \\
\hline Delwel et al. 2019 [53] & $\begin{array}{c}\text { Silness and Loë Plaque } \\
\text { Index } \\
\text { DPSI } \\
\text { Probing pocket depth } \\
\text { (mm) } \\
\text { Mobility grade }\end{array}$ & & $\begin{array}{c}2.0 \\
\text { 3- (IQR }=2.4) \\
\geq 4 \mathrm{~mm} \text { in } 73.8 \% \\
\text { participants } \\
\text { Grade } 2 \text { in } 18.8 \% \\
\text { Grade } 3 \text { in } 5.8 \%\end{array}$ \\
\hline $\begin{array}{c}\text { Gil-Montoya et al. } 2016 \\
\text { [58] }\end{array}$ & Bleeding Index & 50.6 & $67.5^{* * *}$ \\
\hline Hoben et al. 2016 [28] & $\begin{array}{l}\text { Inflamed, swollen or } \\
\text { bleeding gums }\end{array}$ & $1.2 \%$ & $0.8 \%$ \\
\hline Hopcraft et al. 2012 [61] & $\begin{array}{l}\text { Periodontal pocket depth } \\
4 \mathrm{~mm} \text {, periodontal } \\
\text { pocket depth }>6 \mathrm{~mm}\end{array}$ & $35.0 \%$ & $36.0 \%$ \\
\hline Ide et al. 2016 [31] & $\begin{array}{c}\text { Probing depth }>3 \mathrm{~mm} \\
\text { Bleeding on probing } \\
\text { Periodontitis according } \\
\text { to CDC/AAP criteria: } \\
\text { MoD-SeV }\end{array}$ & & $\begin{array}{c}6.7 \% \\
13.6 \% \\
37.3 \\
25.4 \%-1.9 \%\end{array}$ \\
\hline Lee et al. 2013 [62] & Periodontal pocket depth & $1.5 \mathrm{~mm}$ & $1.4 \mathrm{~mm}$ \\
\hline Philip et al. 2012 [64] & $\begin{array}{l}\text { Gingival inflammation: } \\
\text { Minimal: } \\
\text { Light: } \\
\text { Moderate: }\end{array}$ & $\begin{array}{c}22.5 \% \\
36.3 \% \\
32.5 \% \\
6.8 \%\end{array}$ & $\begin{array}{l}13.0 \% \\
21.0 \% \\
56.5 \% \\
13.0 \%\end{array}$ \\
\hline Sumi et al. 2012 [33] & $\begin{array}{l}\text { Gingival Index } \\
\text { Loe-Silness }\end{array}$ & - & 1.2 \\
\hline $\begin{array}{c}\text { Srisilapanan et al. } 2013 \\
\text { [66] }\end{array}$ & $\begin{array}{l}\text { Community Periodontal } \\
\text { Index, highest score: } \\
\text { Normal } \\
\text { Bleeding } \\
\text { Calculus } \\
\text { Pocket depth } 4-5 \mathrm{~mm} \\
\text { Pocket depth } \geq 6 \mathrm{~mm}\end{array}$ & $\begin{array}{l}- \\
- \\
- \\
-\end{array}$ & $\begin{array}{c}9.4 \% \\
1.9 \% \\
34.0 \% \\
30.2 \% \\
24.5 \%\end{array}$ \\
\hline Syrjala et al. 2012 [67] & $\begin{array}{l}\text { Number of teeth with } \\
\text { periodontal pockets }\end{array}$ & & $\begin{array}{l}2.8 \mathrm{AD} \\
2.8 \mathrm{VaD} \\
1.7 \mathrm{OD}\end{array}$ \\
\hline Warren et al. 1997 [43] & $\begin{array}{l}\text { Modified version of } \\
\text { Gingival Index by Silness } \\
\text { and Loe }\end{array}$ & 0.7 & $\begin{array}{cc}1.1 & \mathrm{AD} \\
1.2 & 0.9 \mathrm{OD}\end{array}$ \\
\hline Zenthöfer et al. 2014 [36] & $\begin{array}{l}\text { Periodontitis } \\
\text { Gingival Bleeding Index } \\
\text { Community periodontal } \\
\text { index of treatment needs }\end{array}$ & $\begin{array}{c}73.9 \% \\
40.9 \% \\
2.8\end{array}$ & $\begin{array}{c}100 \% * * * \\
43.8 \% \\
3.4^{* * *}\end{array}$ \\
\hline
\end{tabular}


Table 10. Cont.

\begin{tabular}{clcc}
\hline Study & Outcome Measure & $\begin{array}{c}\text { Gingival Health No } \\
\text { Dementia }\end{array}$ & $\begin{array}{c}\text { Gingival Health } \\
\text { Dementia }\end{array}$ \\
\hline Zenthöfer et al. 2016 [37] & $\begin{array}{l}\text { Gingival Bleeding Index } \\
\text { Community periodontal } \\
\text { index of treatment needs }\end{array}$ & $\begin{array}{c}38.1 \% \\
3.1\end{array}$ & $\begin{array}{c}52.1 \% * \\
3.3\end{array}$ \\
\hline Zenthöfer et al. 2016 [44] & $\begin{array}{c}\text { Community periodontal } \\
\text { index of treatment needs }\end{array}$ & 2.7 & $3.1 * * *$ \\
\hline \multirow{2}{*}{ Zenthöfer et al. 2017 [44] } & $\begin{array}{l}\text { Gingival Bleeding Index } \\
\text { Community periodontal } \\
\text { index of treatment needs }\end{array}$ & $\begin{array}{c}48.8 \% \\
2.7\end{array}$ & $53.8^{* * *}$ \\
\hline
\end{tabular}

AD: Alzheimer's disease; Vad: vascular dementia; OD: other dementia; ${ }^{*} p \leq 0.05 ;{ }^{* *}: p \leq 0.01$; $^{* * *}: p \leq 0.00$.

Zenthöfer $[36,37,44]$ demonstrated that the Gingival Bleeding Index of people with dementia was 43.8 to $53.8 \%$ and confirmed De Souza's results, proving that people with dementia suffer from periodontitis more than people without dementia (community periodontal index of treatment needs was 3.1-3.4 in dementia people and 2.7-2.8 in non dementia people [36,37]).

Nine of the included studies found no significant differences between both groups with regards to oral hygiene $[21,22,27,45,61,62,64-67]$ and five studies demonstrated a higher level of plaque in dementia people $[14,36,43,56,65]$. The Plaque Index by Silness and Loe was 0.7 in the study by Chalmers et al. [13], 2.5 in the study by Gil-Montoya [14] in the elderly with dementia, and 2.0 in the study by Delwel et al. [53]. Sumi et al. [33] showed a Plaque Index by Quigley and Hein of 1.6. O'Leary Plaque Index was significantly higher in dementia people (90.1\%) than in non dementia people [36] (73.3\%). Finally, Ribeiro [65] et al. established that the Oral Hygiene Index by Green and Vermillion is higher in participants with dementia (4.5) than in participants without dementia (2.2). A significantly higher Debris Index in people with moderate to severe dementia was found [43].

Furthermore, edentuloussnes was a condition that affected a large percentage of the elderly, in particular 11.6 to $72.7 \%$ of the elderly with dementia [66,67] and 14.0 to $70 \%$ of the elderly without dementia $[38,45]$. Within partially or totally edentulous participants, denture utilization varied between these percentages: $17.0-81.8 \%$ in normal cognitive people and 5.0 to $100 \%$ in people with cognitive impairment $[27,31]$ (Table 11).

Table 11. Results about dentures and edentulousness.

\begin{tabular}{|c|c|c|c|c|}
\hline Study & $\begin{array}{l}\text { Dentures no } \\
\text { Dementia }\end{array}$ & $\begin{array}{l}\text { Dentures } \\
\text { Dementia }\end{array}$ & $\begin{array}{l}\text { Edentulousness } \\
\text { No Dementia }\end{array}$ & $\begin{array}{c}\text { Edentulousness } \\
\text { Dementia }\end{array}$ \\
\hline Adam et al. 2006 [45] & I & l & $70.0 \%$ & $63.0 \%$ \\
\hline Bomfim et al. 2013 [46] & $20.0 \%$ & $20.0 \%$ & $46.7 \%$ & $40.0 \%$ \\
\hline Chalmers et al. 2003 [13] & $27.6-30.1 \%$ & $20.7-23.3 \%$ & l & l \\
\hline Chapman et al. 1991 [48] & - & $59.0 \%$ & - & $64.7 \%$ \\
\hline Chen et al. 2013 [49] & - & $\begin{array}{c}48.0 \% \mathrm{C} \\
38.9 \% \mathrm{~A} \\
47.1 \% \mathrm{NHR}\end{array}$ & l & l \\
\hline Chu et al. 2015 [38] & $14.0 \%$ & $17.0 \%$ & I & I \\
\hline $\begin{array}{l}\text { De Souza Rolim et al. } \\
2014 \text { [24] }\end{array}$ & $43.3 \%$ & $25.8 \%$ & $43.3 \%$ & $32.3 \%$ \\
\hline Elsig et al. 2013 [56] & l & l & $54.6 \%$ & $62.1 \%$ \\
\hline Eshkoor et al. 2014 [57] & $81.8 \%$ & $86.2 \%$ & l & l \\
\hline Hatipoglu et al. 2011 [27] & $\begin{array}{c}57.0 \% \text { Max } \\
55.0 \% \text { Mand }\end{array}$ & $\begin{array}{c}\text { 97.0\% Max } \\
\text { 100.0\% Mand }\end{array}$ & I & / \\
\hline
\end{tabular}


Table 11. Cont.

\begin{tabular}{ccccc}
\hline Study & $\begin{array}{c}\text { Dentures no } \\
\text { Dementia }\end{array}$ & $\begin{array}{c}\text { Dentures } \\
\text { Dementia }\end{array}$ & $\begin{array}{c}\text { Edentulousness } \\
\text { No Dementia }\end{array}$ & $\begin{array}{c}\text { Edentulousness } \\
\text { Dementia }\end{array}$ \\
\hline Kossioni et al. 2012 [40] & - & $62.9 \%$ & & \\
\hline Nordenram et al. 1996 [42] & $17.0 \%$ & $\begin{array}{c}7.0 \% \mathrm{Mod} \\
5.0 \% \mathrm{Sed}\end{array}$ & $43.0 \%$ & $\begin{array}{c}36.0 \% \mathrm{MoD} \\
45.0 \% \mathrm{SeD}\end{array}$ \\
\hline Ship et al. 1994 [32] & $43.0 \%$ & $40.0-67.0 \%$ & $/$ & $11.6 \%$ \\
\hline $\begin{array}{c}\text { Srisilapapanan et al. } \\
\text { 2013 [66] }\end{array}$ & - & $40.6 \%$ & - & $63.3 \% \mathrm{AD}$ \\
\hline Syrjala et al. 2012 [67] & $73.7 \%$ & $75.5 \% \mathrm{AD}$ & $68.8 \% \mathrm{VaD}$ \\
\hline Warren et al. 1997 [43] & $/$ & $72.2 \% \mathrm{OD}$ & $44.6 \%$ & $40.7 \% \mathrm{OD}$ \\
\hline
\end{tabular}

Mand. Mandibular; Max: Maxillary; MoD: moderate dementia; NHR: nursing home residents; SeD: severe dementia; Vad: vascular dementia; OD: other dementia; *: $p<0.05$.

Data about orofacial pain were extracted from seven of the included studies $[13,24,39,40,45,52]$. The percentage of the elderly with dementia suffering from orofacial pain was higher than that of participants without dementia: 7.4 to $21.7 \%, 6.7$ to $18.5 \%$, respectively. The cross-sectional study by Delwel et al. [53] carefully examined the presence of orofacial pain in the elderly with dementia or MCI, while using the OPS-NVI [12] and self reported pain. The OPS-NVI was $4 \%$ in rest, $10 \%$ during drinking, 19\% during chewing, and 22\% during oral hygiene care. Pain reported by participants with dementia or MCI was $25.7 \%$ overall (Table 12 ).

Table 12. Results about Orofacial pain.

\begin{tabular}{ccc}
\hline Study & Orofacial Pain No Dementia & Orofacial Pain Dementia \\
\hline Adam et al. 2006 [45] & $18.5 \%$ & $7.4 \%$ \\
\hline Chalmers et al. 2003 [13] & $11.2-11.5 \%$ & $18.4-19.0 \%$ \\
\hline Cohen-Mansfield et al. 2002 [52] & - & $60.0 \%$ \\
\hline De Souza Rolim et al. 2014 [24] & $6.7 \%$ & $20.7 \%$ \\
\hline Delwel et al. 2019 [53] & - & $\begin{array}{c}\text { Dementia: } 27.4 \% \\
\text { MCI: } 20.5 \%\end{array}$ \\
\hline Kossioni et al. 2012 [40] & - & $21.7 \%$ \\
\hline & MCI: Mild Cognitive Impairment.
\end{tabular}

The feeling of a dry mouth or xerostomia reached the percentages of $22.0 \%$ in people with dementia and $8.4 \%$ in people without dementia [33] and it was present in $9.1-45 \%$ of the cases and $8.4-20.0 \%$ of the controls [40-43]. Gil-Montoya [58] showed a more drug-induced xerostomia in cases (68.5-72.2\%) than in the controls (36.5\%).

Oral pathology, such as stomatitis and candidiasis, was most common in the cases than in controls. Chu et al. [38] and other authors [24,39,41] reported a percentage of candidiasis of 3.6-30\% for cases and $0.0-5.0 \%$ for controls. Furthermore, $18.1-59.1 \%$ of cases and $0.0-7.4 \%$ of controls showed stomatitis $[13,27,40]$.

In conclusion, with regards to the oral care need, the included studies $[13,47,49,50,61,64,67]$ reported a need of $21 \%$ for cleaning teeth and dentures in the elderly with dementia. Chalmers et al. [47] demonstrated that, with an increasing severity of cognitive impairment, there is also an increase of oral care need: the assistance need for cleaning teeth and dentures in severe dementia was $100.0 \%$, as compared to the assistance need in moderate dementia, which was $57.2 \%$ (teeth) and $97.3 \%$ 
(dentures). Regarding periodontal disease (Table 13), Dintica et al. [25] and Ide et al. [31] found that the mean change (decrease) in MMSE score due to tooth loss was, respectively, -0.94 to 0.37 (nine months follow-up, adjusted for age, sex, and education) and -3.6 to -0.03 (six months follow-up), establishing a significant association between tooth loss and the progression of cognitive impairment. The crude hazard ratio of dementia according to the number of remaining/lost teeth was $1.6[10,34]$. Tiisanoja et al. [68] demonstrated that subjects with pocket depth $\geq 4 \mathrm{~mm}$ had an increased, but not statistically significant, risk of developing Alzheimer's Disease (Relative risk: 1.54). Furthermore, Yoo et al. [35] demonstrated that periodontal treatment lead to a significant decrease in the incidence of dementia. The prospective community-based study by Kato et al. [30] showed that the number of natural teeth was significantly associated with an individual's MMSE score: the percentage of cognitively normal subjects (MMSE scores: 27-30) significantly decreased with a decrease in the number of natural teeth (number of teeth $=5-9$, percentage of participants without cognitive impairment $=26.7 \%$; number of teeth $=15-19$; and, percentage of normal cognitive participants $=44.8 \%$ ). Kato et al. also demonstrated that the use of artificial teeth was associated with cognitive function preservation.

Table 13. Association between periodontal disease and dementia.

\begin{tabular}{|c|c|c|}
\hline Study & Follow-Up Period & $\begin{array}{c}\text { Association between Tooth Loss/Periodontal } \\
\text { Disease and Dementia }\end{array}$ \\
\hline Dintica et al. 2018 [25] & 9 years & $\begin{array}{l}\text { Annual mean change in MMSE by tooth loss: } \\
\qquad-0.94 \text { to }-0.37^{*}\end{array}$ \\
\hline Ide et al. 2016 [31] & 6 months & $\begin{array}{l}\text { Mean change in MMSE by the presence of } \\
\text { periodontal disease: }-3.6 \text { to }-0.03-3.6 \text { to } 0.04 \text { *** }\end{array}$ \\
\hline Kato et al. 2019 [30] & 4 years & $\begin{array}{l}\text { Cochran-Armitage trend test Mann-Whitney U } \\
\text { test Kruskal-Wallis test Spearman's rank } \\
\text { correlation test Student's } t \text {-test }\end{array}$ \\
\hline Lee et al. 2017 [10] & $\begin{array}{l}\text { Index date: date of the first } \\
\text { periodontal disease diagnosis. } \\
\text { Patients were followed until } \\
\text { dementia diagnosis, death, } \\
\text { withdrawal from the NHI or } \\
\text { December 31, } 2012\end{array}$ & $\begin{array}{c}\text { Hazard ratio of having dementia in persons with } \\
\text { periodontal disease } \rightarrow \\
\text { HR: } \\
1.02-1.32 \\
1.01-1.32^{* *}\end{array}$ \\
\hline Takeuchi et al. 2017 [34] & 5 years & $\begin{array}{c}\text { Hazard ratio of having dementia in persons with } \\
\text { 10-19 remaining teeth } \rightarrow \\
\text { HR: } \\
1.45-3.03 \\
1.06-2.46^{* * *}\end{array}$ \\
\hline Tiisanoja et al. 2018 [68] & & $\begin{array}{l}\text { Risk of having } \mathrm{AD} \text { in persons with pocket depth } \\
\qquad 4 \mathrm{~mm} \rightarrow \\
\text { RR: } 1.54\end{array}$ \\
\hline Yoo et al. 2019 [35] & 7 year & $\begin{array}{c}\text { Odds Ratio of having dementia in persons with } \\
\text { 7-12 teeth lost } \rightarrow \\
\text { OR: } 1.272\end{array}$ \\
\hline
\end{tabular}

HR: hazard ratio; *: adjusted for age, sex and education; ${ }^{* *}$ : adjusted for sociodemographic characteristics and

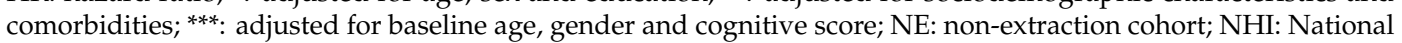
Health Insurance; OR: odds ratio; RR: relative risk; TE: tooth extraction.

\section{Discussion}

The purpose of this systematic review was to examine studies regarding oral health in the elderly with and without dementia and to investigate the relationship between periodontal status and dementia. As reported in a recent review by Delwel et al. [75], the analysis of this study showed no significant differences between the case and controls with regards to the number of present teeth $[13,21,29,43,53,56,61,67,69]$ and to the DMFT Index $[27,38,61,65]$. However, the DMFT categories separately, "decay", "missing", and "filled", give a better indication of disease and treatment need compared to the index, which reports dental caries history as a whole. Coronal and root caries and 
retained roots are most common in people with dementia [21,56,65], and this condition might be explained by cognitive and behavioral deterioration, which reduced the ability to perform routine oral care [76]. In the elderly with dementia saliva flow rates decreases [32], eating habits change (more cariogenic food) [51,56,61,77], and motor skills and coordination worsen [65], which leads to a lower chewing and swallowing efficiency $[56,77]$.

Another important point to be considered is the oppositional and aggressive behavior towards oral care and decreased communication skills, which represent barriers to oral hygiene and assistance. These obstacles could be overcome by performing oral care education to caregivers and by increasing dental checks of the elderly with dementia $[62,78,79]$.

Moreover, the recent observational study by Delwel et al. [53] recorded a significant correlation between the cognitive impairment level and the number of present/missing/restored teeth and retained roots, which suggests that dementia could have a negative impact on oral health.

Concerning oral soft tissues, this study confirmed Delwel's et al. review results [80]: gingival bleeding, periodontal disease, mucosal lesions, and xerostomia were found at higher rates in participants with cognitive impairment. Dry mouth was more common in the elderly, who used medication or had radiotherapy history (head and neck) or autoimmune disease [32].

Approximately the same percentage of the elderly either with or without dementia wore dentures [13,42]. However, people with an advanced dementia degree showed a lower use of dentures, as compared to people with moderate dementia, because of the musculature, salivary flow decrease, and lower tolerance of dentures.

The deterioration of verbal communication skills and the higher prevalence of oral disease might cause a higher suffering to people with dementia, due to orofacial pain $[45,50,52,53]$. In this systematic review, the new information included concern the potential role of periodontal disease as a risk factor for developing cognitive impairment. In fact, all of the studies included in Table 10 demonstrated an association between teeth loss due to periodontal disease and the onset of dementia $[10,25,30,31,34,35,60]$. This association is based on biological mechanisms: subjects with periodontal disease or antibodies to periodontal bacterial flora show an increased systemic proinflammatory state, which lead to an increase of the cognitive decline rate [31]. According to Yoo et al., the more teeth are lost, the higher incidence of dementia is. The author also stated that monitoring cognitive status in patients with extensive teeth loss could lead to an early diagnosis of dementia. The significant association between the number of natural teeth and the MMSE score was also confirmed in the study by Kato et al., which suggest that the use of artificial teeth could help to preserve the cognitive function.

\section{Strengths and Limitations}

The most important limitation of this study is the result of the quality assessment of the articles, since more than half of the included studies have a score equal or below 5 . The number of high quality studies was low and no homogeneity can be found. For this reason, it was impossible to perform a meta-analysis. In the included studies, several different measures were used to evaluate oral health status and some studies did not distinguish between the elderly with and without dementia.

The main strength of this review is the systematic approach and involvement of a multidisciplinary team (dentists, neuropsychologist, pain specialist). In addition, in almost all of the studies reviewed, oral examinations are structured and standardized, and were carried out by dentists.

\section{Conclusions}

The elderly with dementia show a higher level of plaque, coronal and root caries, retained roots, gingival, and periodontal disease. Further attention is needed regarding orofacial pain, which is very common in dementia people. Poor oral health within this group could be increased by the reduction of submandibular salivary flow, deterioration of cognitive functions, motor and communication skills, and aggressive behavior. Caregivers should be educated and dentist's checks in nursing homes should be enhanced in order to improve the oral health status of the elderly with dementia. Our contribution 
highlights the relationship between periodontal disease and dementia. Teeth loss due to periodontal disease increases the risk of cognitive function deterioration. However, the specific mechanisms of this association need further investigation.

Author Contributions: Conceptualization, D.L. and G.M.; methodology, F.D.V.; validation A.L. and F.C.; formal analysis, D.D.S.; investigation, G.M.; data curation, D.L.; writing-original draft preparation, G.M.; writing-review and editing, D.L.; visualization, F.C.; supervision, M.P.

Funding: This research received no external funding.

Conflicts of Interest: The authors declare no conflict of interest.

\section{References}

1. National Institute on Aging; National Institutes of Health, U.S.; Department of Health and Human Services. Global Health and Aging; World Health Organisation: Geneva, Switzerland, 2011.

2. Lopez, R.; Smith, P.C.; Göstemeyer, G.; Schwendicke, F. Ageing, dental caries and periodontal diseases. J. Clin. Periodontol. 2017, 44 (Suppl. 18), S145-S152. [CrossRef] [PubMed]

3. Nangle, M.R.; Riches, J.; Grainger, S.A.; Manchery, N.; Sachdev, P.S.; Henry, J.D. Oral Health and Cognitive Function in Older Adults: A systematic Review. Gerodontology 2019, 65, 659-672. [CrossRef] [PubMed]

4. Wu, B.; Fillenbaum, G.G.; Plassman, B.L.; Guo, L. Association Between Oral Health and Cognitive Status: A Systematic Review. J. Am. Geriatr. Soc. 2016, 64, 739-751. [CrossRef] [PubMed]

5. Moritz, D.J.; Kasl, S.V.; Berkman, L.F. Cognitive Functioning and the Incidence of Limitations in Activities of Daily Living in an Elderly Community Sample. Am. J. Epidemiol. 1995, 141, 41-49. [CrossRef] [PubMed]

6. Ship, J.A.; De Carli, C.; Friedland, R.P.; Baum, B.J. Diminished submandibular salivary flow in dementia of the Alzheimer type. J. Gerontol. 1990, 45, M61-M66. [CrossRef] [PubMed]

7. Ship, J.A.; Pillemer, S.R.; Baum, B.J. Xerostomia and the geriatric patient. J. Am. Geriatr. Soc. 2002, 50, 535-543. [CrossRef] [PubMed]

8. Silva, M.; Hopcraft, M.; Morgan, M. Dental caries in Victorian nursing homes. Aust. Dent. J. 2014, 59, 321-328. [CrossRef]

9. Martande, S.S.; Pradeep, A.R.; Singh, S.P.; Kumari, M.; Suke, D.K.; Raju, A.P.; Naik, S.B.; Singh, P.; Guruprasad, C.N.; Chatterji, A. Periodontal Health Condition in Patients With Alzheimer's Disease. Am. J. Alzheimers Dis. Other Demen. 2014, 29, 498-502. [CrossRef]

10. Lee, Y.T.; Lee, H.C.; Hu, C.J.; Huang, L.K.; Chao, S.P.; Lin, C.P.; Su, E.C.; Lee, Y.C.; Chen, C.C. Periodontitis as a Modifiable Risk Factor for Dementia: A Nationwide Population-Based Cohort Study. Am. Geriatr. Soc. 2017, 65, 301-305. [CrossRef]

11. Ewan, V.; Staines, K. Diagnosis and management of oral mucosal lesions in older people: A review. Rev. Clin. Gerontol. 2008, 18, 115-128. [CrossRef]

12. De Vries, M.W.; Visscher, C.; Delwel, S.; van der Steen, J.T.; Pieper, M.J.C.; Scherder, E.J.A.; Achterberg, W.P.; Lobbezoo, F. Orofacial Pain during Mastication in People with Dementia: Reliability Testing of the Orofacial Pain Scale for Non-Verbal Individuals. Behav. Neurol. 2016, 2016, 3123402. [CrossRef] [PubMed]

13. Chalmers, J.M.; Carter, K.D.; Spencer, A.J. Oral diseases and conditions in community-living older adults with and without dementia. Spec. Care Dent. 2003, 23, 7-17. [CrossRef] [PubMed]

14. Gil-Montoya, J.A.; Sanchez-Lara, I.; Carnero-Pardo, C.; Fornieles-Rubio, F.; Montes, J.; Barrios, R.; Gonzalez-Moles, M.A.; Bravo, M. Oral Hygiene in the Elderly with Different Degrees of Cognitive Impairment and Dementia. J. Am. Geriatr. Soc. 2017, 65, 642-647. [CrossRef]

15. Cerutti-Kopplin, D.; Feine, J.; Padilha, D.M.; de Souza, R.F.; Ahmadi, M.; Rompré, P.; Booij, L.; Emami, E. Tooth Loss Increases the Risk of Diminished Cognitive Function: A Systematic Review and Meta-analysis. JDR Clin. Trans. Res. 2016, 1, 10-19. [CrossRef] [PubMed]

16. Noble, J.M.; Scarmeas, N.; Papapanou, P.N. Poor Oral Health as a Chronic, Potentially, Modifiable Dementia Risk Factor: Review of Literature. Curr. Neurol. Neurosci. Rep. 2013, 13, 384. [CrossRef] [PubMed]

17. Liberati, A.; Altman, D.G.; Tetzlaff, J.; Mulrow, C.; Gøtzsche, P.C.; Ioannidis, J.P.; Clarke, M.; Devereaux, P.J.; Kleijnen, J.; Moher, D. The PRISMA statement for reporting systematic reviews and meta-analyses of studies that evaluate health care interventions: Explanation and elaboration. J. Clin. Epidemiol. 2009, 62, e1-e34. [CrossRef] [PubMed] 
18. Wells, G.A.; Shea, B.; O'Connell, D.; Petersen, J.; Welch, V.; Losos, M.; Tugwell, P. The Newcastle-Ottawa Scale (NOS) for Assessing the Quality of Nonrandomized Studies in Meta-Analyses; Department of Epidemiology and Community Medicine, University of Ottawa, Canada: Ottawa, ON, Canada, 2012.

19. Hoeksema, A.R.; Peters, L.L.; Raghoebar, G.M.; Meijer, H.J.A.; Vissink, A.; Visser, A. Oral health status and need for oral care of care-dependent indwelling elderly: From admission to death. Clin. Oral Investig. 2017, 21, 2189-2196. [CrossRef] [PubMed]

20. Luo, J.; Wu, B.; Zhao, Q.; Guo, Q.; Meng, H.; Yu, L.; Zheng, L.; Hong, Z.; Ding, D. Association between tooth loss and cognitive function among 3063 Chinese older adults: A community-based study. PLoS ONE 2015, 10, e0120986. [CrossRef]

21. Chalmers, J.M.; Carter, K.D.; Spencer, A.J. Caries incidence and increments in community-living older adults with and without dementia. Gerodontology 2002, 19, 80-94. [CrossRef]

22. Chalmers, J.M.; Carter, K.; Spencer, A. Oral health of Adelaide nursing home residents: Longitudinal study. Aust. J. Ageing 2004. [CrossRef]

23. Chen, X.; Shuman, S.K.; Hodges, J.S.; Gatewood, L.C.; Xu, J. Patterns of tooth loss in older adults with and without dementia: A retrospective study based on a Minnesota cohort. J. Am. Geriatr. Soc. 2010, 58, 2300-2307. [CrossRef] [PubMed]

24. De Souza Rolim, T.; Fabri, G.M.; Nitrini, R.; Anghinah, R.; Teixeira, M.J.; de Siqueira, J.T.; Cestari, J.A.; de Siqueira, S.R. Oral infections and orofacial pain in Alzheimer's disease: A case-control study. J. Alzheimers Dis. 2014, 38, 823-829. [CrossRef] [PubMed]

25. Dintica, C.S.; Rizzuto, D.; Marseglia, A.; Kalpouzos, G.; Welmer, A.K.; Wårdh, I.; Bäckman, L.; Xu, W. Tooth loss is associated with accelerated cognitive decline and volumetric brain differences: A population-based study. Neurobiol. Aging 2018, 67, 23-30. [CrossRef] [PubMed]

26. Ellefsen, B.; Holm-Pedersen, P.; Morse, D.E.; Schroll, M.; Andersen, B.B.; Waldemar, G. Assessing caries increments in elderly patients with and without dementia: A one-year follow-up study. J. Am. Dent. Assoc. 2009, 140, 1392-1400. [CrossRef]

27. Hatipoglu, M.G.; Kabay, S.C.; Güven, G. The clinical evaluation of the oral status in Alzheimer-type dementia patients. Gerodontology 2011, 28, 302-306. [CrossRef]

28. Hoben, M.; Poss, J.W.; Norton, P.G.; Estabrooks, C.A. Oral/dental items in the resident assessment instrument-minimum data set 2.0 lack validity: Results of a retrospective, longitudinal validation study. Popul. Health Metr. 2016, 14, 36. [CrossRef]

29. Jones, J.A.; Lavallee, N.; Alman, J.; Sinclair, C.; Garcia, R.I. Caries incidence in patients with dementia. Gerodontology 1993, 10, 76-82. [CrossRef]

30. Kato, H.; Takahashi, Y.; Iseki, C.; Igari, R.; Sato, H.; Sato, H.; Koyama, S.; Tobita, M.; Kawanami, T.; Iino, M.; et al. Tooth Loss-associated Cognitive Impairment in the Elderly: A Community-based Study in Japan. Intern. Med. 2019, 58, 1411-1416. [CrossRef]

31. Ide, M.; Harris, M.; Stevens, A.; Sussams, R.; Hopkins, V.; Culliford, D.; Fuller, J.; Ibbett, P.; Raybould, R.; Thomas, R.; et al. Periodontitis and cognitive decline in Alzheimer's disease. PLoS ONE 2016, 11, e0151081. [CrossRef]

32. Ship, J.A.; Puckett, S.A. Longitudinal study on oral health in subjects with Alzheimer's disease. J. Am. Geriatr. Soc. 1994, 42, 57-63. [CrossRef]

33. Sumi, Y.; Ozawa, N.; Michiwaki, Y.; Washimi, Y.; Toba, K. Oral conditions and oral management approaches in mild dementia patients. Nihon Ronen Igakkai Zasshi 2012, 49, 90-98. [CrossRef] [PubMed]

34. Takeuchi, K.; Ohara, T.; Furuta, M.; Takeshita, T.; Shibata, Y.; Hata, J.; Yoshida, D.; Yamashita, Y.; Ninomiya, T. Tooth Loss and Risk of Dementia in the Community: The Hisayama Study. J. Am. Geriatr. Soc. 2017, 65, e95-e100. [CrossRef] [PubMed]

35. Yoo, J.J.; Yoon, J.H.; Kang, M.J.; Kim, M.; Oh, N. The effect of missing teeth on dementia in older people: A nationwide population-based cohort study in South Korea. BMC Oral Health 2019, 19, 61. [CrossRef] [PubMed]

36. Zenthöfer, A.; Schröder, J.; Cabrera, T.; Rammelsberg, P.; Hassel, A.J. Comparison of oral health among older people with and without dementia. Community Dent. Health 2014, 31, 27-31.

37. Zenthöfer, A.; Cabrera, T.; Rammelsberg, P.; Hassel, A.J. Improving oral health of institutionalized older people with diagnosed dementia. Aging Ment. Health 2016, 20, 303-308. [CrossRef] 
38. Chu, C.H.; Ng, A.; Chau, A.M.; Lo, E.C. Oral health status of elderly Chinese with dementia in Hong Kong. Oral Health Prev. Dent. 2015, 13, 51-57. [CrossRef]

39. Rolim Tde, S.; Fabri, G.M.; Nitrini, R.; Anghinah, R.; Teixeira, M.J.; Siqueira, J.T.; Cesari, J.A.; Siqueira, S.R. Evaluation of patients with Alzheimer's disease before and after dental treatment. Arq. Neuropsiquiatr. 2014, 72, 919-924. [CrossRef]

40. Kossioni, A.E.; Kossionis, G.E.; Polychronopoulou, A. Oral health status of elderly hospitalised psychiatric patient. Gerodontology 2012, 29, 272-283. [CrossRef]

41. Leal, S.C.; Bittar, J.; Portugal, A.; Falcão, D.P.; Faber, J.; Zanotta, P. Medication in elderly people: Its influence on salivary pattern, signs and symptoms of dry mouth. Gerodontology 2010, 27, 129-133. [CrossRef]

42. Nordenram, G.; Ryd-Kjellen, E.; Johansson, G.; Nordstrom, G.; Winblad, B. Alzheimer's disease, oral function and nutritional status. Gerodontology 1996, 13, 9-16. [CrossRef]

43. Warren, J.J.; Chalmers, J.M.; Levy, S.M.; Blanco, V.L.; Ettinger, R.L. Oral health of persons with and without dementia attending a geriatric clinic. Spec. Care Dent. 1997, 17, 47-53. [CrossRef] [PubMed]

44. Zenthöfer, A.; Baumgart, D.; Cabrera, T.; Rammelsberg, P.; Schröder, J.; Corcodel, N.; Hassel, A.J. Poor dental hygiene and periodontal health in nursing home residents with dementia: An observational study. Odontology 2017, 105, 208-213. [CrossRef] [PubMed]

45. Adam, H.; Preston, A.J. The oral health of individuals with dementia in nursing homes. Gerodontology 2006, 23, 99-105. [CrossRef] [PubMed]

46. Bomfim, F.M.S.; Chiari, B.M.; Roque, F.P. Fatores associados a sinais sugestivos de disfagia orofaríngea em idosas institucionalizadas. CoDAS 2013, 25, 154-163. [CrossRef] [PubMed]

47. Chalmers, J.M.; Hodge, C.; Fuss, J.M.; Spencer, A.J.; Carter, K.D. The prevalence and experience of oral diseases in Adelaide nursing home residents. Aust. Dent. J. 2002, 47, 123-130. [CrossRef]

48. Chapman, P.J.; Shaw, R.M. Normative dental treatment needs of Alzheimer patients. Aust. Dent. J. 1991, 36, 141-144. [CrossRef]

49. Chen, X.; Clark, J.J.; Naorungroj, S. Oral health in older adults with dementia living in different environments: A propensity analysis. Spec. Care Dent. 2013, 33, 239-247. [CrossRef]

50. Chen, X.; Clark, J.J.; Naorungroj, S. Oral health in nursing home residents with different cognitive statuses. Gerodontology 2013, 30, 49-60. [CrossRef]

51. Chen, X.; Clark, J.J.; Chen, H.; Naorungroj, S. Cognitive impairment, oral self-care function and dental caries severity in community-dwelling older adults. Gerodontology 2015, 32, 53-61. [CrossRef]

52. Cohen-Mansfield, J.; Lipson, S. The underdetection of pain of dental etiology in persons with dementia. Am. J. Alzheimers Dis. Other Demen. 2002, 17, 249-253. [CrossRef]

53. Delwel, S.; Scherder, E.J.A.; de Baat, C.; Binnekade, T.T.; van der Wouden, J.C.; Hertogh, C.M.P.M.; Maier, A.B.; Perez, R.S.G.M.; Lobbezoo, F. Orofacial pain and its potential oral causes in older people with mild cognitive impairment or dementia. J. Oral Rhabilit. 2019, 46, 23-32. [CrossRef] [PubMed]

54. Ellefsen, B.; Holm-Pedersen, P.; Morse, D.E.; Schroll, M.; Andersen, B.B.; Waldemar, G. Caries prevalence in older persons with and without dementia. J. Am. Geriatr. Soc. 2008, 56, 59-67. [CrossRef] [PubMed]

55. Ellefsen, B.S.; Morse, D.E.; Waldemar, G.; Holm-Pedersen, P. Indicators for root caries in Danish persons with recently diagnosed Alzheimer's disease. Gerodontology 2012, 29, 194-202. [CrossRef] [PubMed]

56. Elsig, F.; Schimmel, M.; Duvernay, E.; Giannelli, S.V.; Graf, C.E.; Carlier, S.; Herrmann, F.R.; Michel, J.P.; Gold, G.; Zekry, D.; et al. Tooth loss, chewing efficiency and cognitive impairment in geriatric patients. Gerodontology 2015, 32, 149-156. [CrossRef]

57. Eshkoor, S.A.; Hamid, T.A.; Nudin, S.S.; Mun, C.Y. Association between dentures and the rate of falls in dementia. Med. Devices 2014, 7, 225-230. [CrossRef]

58. Gil-Montoya, J.A.; Barrios, R.; Sánchez-Lara, I.; Carnero-Pardo, C.; Fornieles-Rubio, F.; Montes, J.; Gonzalez-Moles, M.A.; Bravo, M. Prevalence of drug-induced xerostomia in older adults with cognitive impairment or dementia: An observational study. Drugs Aging 2016, 33, 611-618. [CrossRef]

59. Kossioni, A.E.; Kossionis, G.E.; Polychronopoulou, A. Self-reported oral complaints in older mentally ill patient. Geriatr. Gerontol. Int. 2013, 13, 358-364. [CrossRef]

60. Furuta, M.; Komiya-Nonaka, M.; Akifusa, S.; Shimazaki, Y.; Adachi, M.; Kinoshita, T.; Kikutani, T.; Yamashita, Y. Interrelationship of oral health status, swallowing function, nutritional status, and cognitive ability with activities of daily living in Japanese elderly people receiving home care services due to physical disabilities. Community Dent. Oral Epidemiol. 2013, 41, 173-181. [CrossRef] 
61. Hopcraft, M.S.; Morgan, M.V.; Satur, J.G.; Wright, F.A.C. Edentulism and dental caries in Victorian nursing homes. Gerodontology 2012, 29, e512-e519. [CrossRef]

62. Lee, K.H.; Wu, B.; Plassman, B.L. Cognitive function and oral health-related quality of life in older adults. J. Am. Geriatr. Soc. 2013, 61, 1602-1607. [CrossRef]

63. Minakuchi, S.; Takaoka, S.; Shimoyama, K.; Uematsu, H. Factors affecting denture use in some institutionalized elderly people. Spec. Care Dent. 2006, 26, 101-105. [CrossRef] [PubMed]

64. Philip, P.; Rogers, C.; Kruger, E.; Tennant, M. Oral hygiene care status of elderly with dementia and in residential aged care facilities. Gerodontology 2012, 29, e306-e311. [CrossRef] [PubMed]

65. Ribeiro, G.R.; Costa, J.L.; Ambrosano, G.M.; Garcia, R.C. Oral health of the elderly with Alzheimer's diseas. Oral Surg. Oral Med. Oral Pathol. Oral Radiol. 2012, 114, 338-343. [CrossRef] [PubMed]

66. Srisilapanan, P.; Jai-Ua, C. Oral health status of dementia patients in Chiang Mai Neurological Hospital. J. Med. Assoc. Thai 2013, 96, 351-357.

67. Syrjälä, A.-M.H.; Ylöstalo, P.; Ruoppi, P.; Komulainen, K.; Hartikainen, S.; Sulkava, R.; Knuuttila, M. Dementia and oral health among subjects aged 75 years or older. Gerodontology 2012, 29, 36-42. [CrossRef]

68. Tiisanoja, A.; Syrjala, A.M.; Tertsonen, M.; Komulainen, K.; Pesonen, P.; Knuuttila, M.; Hartikainen, S.; Ylostalo, P. Oral disease and inflammatory burden and Alzheimer's disease among subjects aged 75 years or older. Spec. Care Dent. 2019, 39, 158-165. [CrossRef]

69. Fjeld, K.G.; Mowe, M.; Eide, H.; Willumsen, T. Effect of electric toothbrush on residents' oral hygiene: A randomized clinical trial in nursing homes. Eur. J. Oral Sci. 2014, 122, 142-148. [CrossRef]

70. American Psychiatric Association. Diagnostic and Statistical Manual of Mental Disorders, 4th ed.; Text. Revision DSM-IV-TR; American Psychiatric Association: Arlington, VA, USA, 2000.

71. World Health Organization. ICD-10 International Statistical Classification of Diseases and Related Health Problems; WHO: Geneva, Switzerland, 2008.

72. McKhann, G.M.; Knopman, D.S.; Chertkow, H.; Hyman, B.T.; Jack, C.R., Jr.; Kawas, C.H.; Klunk, W.E.; Koroshetz, W.J.; Manly, J.J.; Mayeux, R.; et al. The diagnosis of dementia due to Alzheimer's disease: Recommendations from the National Institute on Aging-Alzheimer's association workgroups on diagnostic guidelines for Alzheimer's disease. Alzheimers Dement. 2011, 7, 263-269. [CrossRef]

73. Folstein, M.F.; Folstein, S.E.; McHugh, P.R. Mini-mental state. J. Psychiatr. Res. 1975, 12, 189-198. [CrossRef]

74. Morris, J.C. The clinical dementia rating (CDR): Current version and scoring rules. Neurology 1993, 43, 2412-2414. [CrossRef]

75. Delwel, S.; Binnekade, T.T.; Perez, R.S.; Hertogh, C.M.; Scherder, E.J.; Lobbezzoo, F. Oral health and Orofacial pain in older people with dementia: A systematic review with focus on dental hard tissues. Clin. Oral Investig. 2017, 21, 17-32. [CrossRef] [PubMed]

76. Weijenberg, R.A.F.; Delwel, S.; Ho, B.V.; van der Maarel-Wierink, C.D.; Lobbezoo, F. Mind your teeth-The relationship between mastication and cognition. Gerodontology 2019, 36, 2-7. [CrossRef] [PubMed]

77. Delwel, S.; Scherder, E.J.A.; Perez, R.S.G.M.; Hertogh, C.M.P.M.; Maier, A.B.; Lobbezoo, F. Oral function of older people with mild cognitive impairment or dementia. J. Oral Rehabilit. 2018, 45, 990-997. [CrossRef] [PubMed]

78. Jablonski, R.A.; Therrien, B.; Mahoney, E.K.; Kolanowski, A.; Gabello, M.; Brock, A. An intervention to reduce care-resistant behavior in persons with dementia during oral hygiene: A pilot study. Spec. Care Dent. 2011, 31, 77-87. [CrossRef] [PubMed]

79. Willumsen, T.; Karlsen, L.; Naess, R.; Bjørntvedt, S. Are the barriers to good oral hygiene in nursing homes within the nurses or the patients? Gerodontology 2012, 29, e748-e755. [CrossRef] [PubMed]

80. Delwel, S.; Binnekade, T.T.; Perez Roberto, S.G.; Hertogh, C.M.P.M.; Scherder, E.J.A.; Lobbezzoo, F. Oral hygiene and oral health in older people with dementia: A comprehensive review with focus on oral soft tissues. Clin. Oral Investig. 2018, 22, 93-108. [CrossRef] [PubMed]

(C) 2019 by the authors. Licensee MDPI, Basel, Switzerland. This article is an open access article distributed under the terms and conditions of the Creative Commons Attribution (CC BY) license (http://creativecommons.org/licenses/by/4.0/). 